
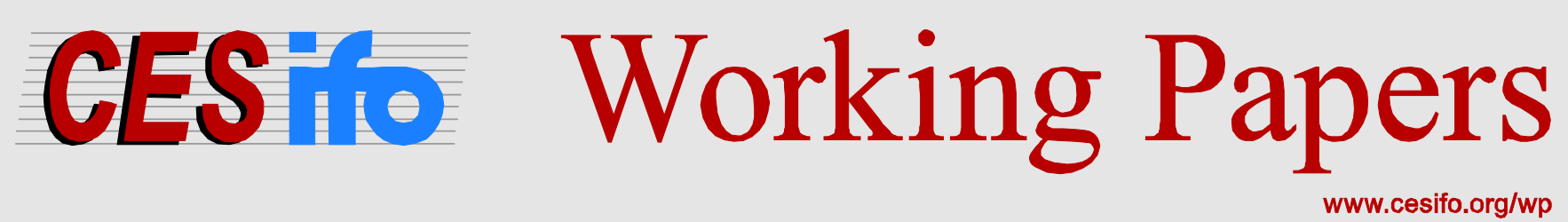

\title{
Dynamic Entry in Vertically Differentiated Markets
}

\author{
Raphael A. Auer \\ Philip Sauré
}

\section{CESIFO WORKING PAPER NO. 6130 \\ CATEGORY 11: INDUSTRIAL ORGANISATION \\ OCTOBER 2016}
An electronic version of the paper may be downloaded
- from the SSRN website: Www.SSRN.com
- from the RePEc website: Www.RePEc.org
- from the CESifo website: www.CESifo-group.org/wp




\title{
Dynamic Entry in Vertically Differentiated Markets
}

\begin{abstract}
We develop a model of vertical innovation in which firms incur a market entry cost and choose a unique level of quality. Once established, firms compete for market shares, selling to consumers with heterogeneous tastes for quality. The equilibrium of the pricing game exists and is unique within our setup. Exogenous productivity growth induces firms to enter the market sequentially at the top end of the quality spectrum. A central feature of the model is that optimization problems of consecutive entrants are self-similar so that new firms enter in constant time-intervals and choose qualities that are a constant fraction higher than incumbent qualities. The asymmetries of quality choice, which inevitably arise because the quality spectrum has top and a bottom, is thus overcome by sequential entry. Our main contribution lies in handling these asymmetries.
\end{abstract}

JEL-Codes: A110, D430, L110, L130, O400.

Keywords: vertical differentiation, product quality, non-homogenous preferences, natural monopoly, endogenous growth, quality ladders.

\author{
Raphael A. Auer \\ Bank of International Settlement \\ Centralbahnplatz 2 \\ Switzerland - 4002 Besel \\ raphael.auer@bis.org
}

\author{
Philip Sauré* \\ Swiss National Bank \\ Börsenstrasse 15 \\ Switzerland - 8001 Zurich \\ philip.saure@snb.ch
}

*corresponding author

September 2016

We would like to thank two anonymous referees, the editor Xavier Vives as well as Thomas Chaney, Sylvain Chassang, Juan Carlos Hallak, Philip Reny, Eric Verhoogen, and Josef Zweimüller and seminar participants at the Federal Reserve Bank of Philadelphia, the 2010 meetings of the European Economic Association in Glasgow, University of Zurich, and the CEPR conference on product quality in international trade for valuable discussions. This paper was previously circulated under the title "Spatial Competition in Quality". The views expressed in this paper are the authors.views and do not necessarily represent those of the BIS or the SNB. 


\section{Introduction}

Quality is one of the main dimensions through which firms differentiate their products. ${ }^{1}$ An important feature of such differentiation in quality - or vertical differentiation - is that all individuals agree on the ranking of varieties. This feature of individual demand has important effects for the equilibrium market structure: supply is naturally concentrated to a small set of firms under a wide set of specifications. The existing literature has therefore focused on cases where either natural monopolies (Shaked and Sutton (1984) and Sutton (2007a and 2007b)) or duopolies prevail (Mussa and Rosen (1978), Gabszewicz and Thisse (1979), Anderson et al. (1989), Champsaur and Rochet (1989) and Motta (1993)) or where oligopolies arise with a bounded number of active firms (Shaked and Sutton (1983 and 1987), Gabszewicz and Thisse (1980) and Lahmandi-Ayed (2000 and 2004)). ${ }^{2}$

Complementing the existing literature, the main interest of the present paper lies in analyzing markets with 'long quality ladders', i.e., markets in which many different quality levels are simultaneously supplied. We thus analyze a setup in which markets accommodate a potentially unbounded number of active firms. In particular, we analyze endogenous quality choice under costly entry in vertically differentiated markets, where each quality is consumed by a different set of consumers according to their valuation of quality. Exogenous productivity growth makes ever-higher qualities affordable so that firms enter the market sequentially at the top end of the quality spectrum. We specify the conditions under which the entry problem of each new entrant is replicated. Our central result is that new qualities improve upon existing qualities by a fixed proportion.

The building block of our analysis is a framework based on Mussa and Rosen (1978), Gabszewicz and Thisse (1979), Bresnahan (1980), and Shaked and Sutton (1982). The model features heterogeneous consumers and a potentially unbounded number of firms, each of which holds the blueprint of a unique quality of an otherwise homogeneous good. All consumers appreciate quality but differ in the degree of this valuation and, thus, in

\footnotetext{
${ }^{1}$ Bils and Klenow (2001) and Broda and Romalis (2009) show empirically that quality differentiation is prevalent in most categories of goods and that for these categories, households of different income consume goods of different quality. The international trade literature has also documented that most industries are characterized by a high degree of vertical specialization. Richer countries both export and import goods of higher quality (see Schott (2004), Hummels and Klenow (2005), Hallak (2006), Hallak and Schott (2011), and Khandewal (2010)).

${ }^{2}$ See Chapter 8 in Anderson et al. (1992) for a comprehensive review of the earlier part of the literature.
} 
their willingness to pay for quality. A key assumption concerning technology is that the marginal production cost is convex in quality, which guarantees the survival of many firms. ${ }^{3}$ In the unique equilibrium, the market is perfectly segmented: the top-quality (bottom) firm supplies consumers with the highest (lowest) valuations, and intermediate firms supply consumers with intermediate valuations accordingly.

Our analysis proceeds in two steps. The first focuses on Bertrand-type price competition for given qualities of an arbitrary number of firms. The prior literature has shown the existence and uniqueness of the Bertrand-type price game. In addition, we provide closed form solutions for firms' prices and profits under a specific, regular distribution of qualities, which is shown to emerge in equilibrium in the second step of our analysis.

The second and main step of our analysis endogenizes firms' quality choices. Firms can incur a fixed cost to improve upon existing qualities, in which case they are granted a perpetual patent to produce their particular quality. Exogenously growing productivities reduce entry and production costs at equal rates. We prove that in this setup, there is a dynamic equilibrium in which each new entrant chooses a quality that is a constant percentage higher than the incumbent technology leader. We then analyze the resulting degree of product market competition as a function of market size and production costs. Specifically, larger markets and lower entry costs induce more frequent firm entry and moredensely supplied qualities because sales and profits lead to a more rapid recovery of setup costs. Surprisingly, an equal percentage increase in the marginal cost of production for all firms is associated with a more densely supplied market. This result holds because markups are proportional to costs. Thus, when production costs rise for all firms, profits actually increase for any given quality spacing. Because excess profits cannot exist in equilibrium, the market must exhibit denser quality spacing and tougher competition.

We regard the main contribution of this paper as technical in that it solves the entry game for the case of monopolistic competition in vertically differentiated markets. The technical difficulties of endogenous quality choice are solved by analyzing a dynamic setup with sequential entry. While sequential market entry of firms obviously generates technical

\footnotetext{
${ }^{3}$ We are not the first to note that a globally convex marginal cost schedule with respect to quality guarantees that a large number of firms can co-exist in equilibrium (see Bresnahan (1980 and 1987), Gabszewicz and Thisse (1980), Shaked and Sutton (1982), and, in particular, the discussion in Anderson et al. (1992)). Similar to the current paper is Zweimüller and Brunner (2005), who study innovation incentives in a model that features many firms supplying different qualities.
} 
difficulties, it also enables us to overcome the problems that unavoidably arise when the choice of quality is endogenous: the quality spectrum has a top end and a bottom end and the respective border conditions generate asymmetries among firms. Handling these resulting analytical difficulties constitutes our main technical contribution.

More specifically, we establish the existence of a quasi-stationary, periodic equilibrium in which each firm enters the industry as the technological leader and successively transits through the product cycle as it becomes superseded by further innovators. The advantage of such a dynamic entry game is that we only have to analyze the entry problem of one firm at a time, since the quality choice and product cycle of all subsequent entrants are isomorphic. We thus circumvent problems that arise in a simultaneous-entry game (see, e.g. Vogel (2008) for the case of horizontal differentiation).

We argue that the case of a large number of competitors in vertically differentiated industries is empirically important, and this case is the particular focus of our paper. For example, empirical studies in the field of international trade document that most manufacturing industries are characterized by many coexisting firms that supply the same good at different levels of quality. ${ }^{4}$ Moreover, given the prominence of quality and non-homothetic preferences in many applied fields such as economic growth (e.g., see Aghion and Howitt (1992)), international trade (e.g., see Fajgelbaum et al. (2011)), or international macroeconomics (e.g., see Bems and Di Giovanni (2014)), we believe that rigorously modeling how firms compete monopolistically in quality constitutes value added per se.

By incorporating consumer heterogeneity as a key feature, our model also relates to the literature on income disparity and quality differentiation. For example, Auer, Chaney and Sauré (2014) apply the setup developed in the current paper to examine how income disparities affect pricing to market decisions and endogenous markups for goods that are differentiated by quality. Our setup is also related to the literature on the connection between income disparity and innovation. ${ }^{5}$

In addition, we argue that our methodological approach of analyzing firm entry in a dynamically evolving environment opens a novel perspective on some of the central ques-

\footnotetext{
${ }^{4}$ See, in particular, Khandelwal (2010) and Kugler and Verhoogen (2010), but also Baldwin and Harrigan (2011), Johnson (2012), Verhoogen (2008), and Hallak and Schott (2009). In addition, industry-level studies document the frequent coexistence of a technological leader and multiple lagging firms (see, for example, Aizcorbe and Kortum (2005)).

${ }^{5}$ See Foellmi and Zweimueller (2006) for a contribution and a literature review.
} 
tions in industrial organization, such as the relation between market size and markups (e.g., see Zhelobodko et al. (2012)) or the optimality of the number of firms in a decentralized economy (see, for example, Lancaster (1966), Dixit and Stiglitz (1977), Vives (2001), and Dhingra and Morrow (2014)). The present paper offers a tool to analyze these important questions from a dynamic perspective. As such, our approach adds to models that have an intrinsically dynamic aspect, e.g., those models analyzing efficiency under sequential entry (e.g., see Vives (1988) or Anderson and Engers (2001)).

On the technical side, our analysis adds to the sizeable literature on quality competition based on Gabszewicz and Thisse (1979), Mussa and Rosen (1978), Bresnahan (1980), and Shaked and Sutton (1982 and 1983). Most of this literature focuses on vertically differentiated markets in which natural oligopolies prevail (for a survey of this literature, see Anderson et al. (1992)). A key assumption in these previous contributions is that the marginal production costs increase only moderately with quality, which enables highquality firms to price low-quality competitors out of the market. Our work, in contrast, complements the literature of setups with the potential to accommodate larger numbers of firms (Bresnahan (1980, 1987), Gabszewicz and Thisse (1980), Shaked and Sutton (1982), Lahmandi-Ayed (2000 and 2004)) by analyzing endogenous quality choice under costly entry.

The remainder of this paper is structured as follows. In Section 2, we develop a theoretical model of competition in quality and examine firms' pricing behavior. We then characterize free entry decisions and quasi-stationary equilibria in Section 3, which we analyze in Section 4. Finally, Section 5 concludes.

\section{A Model of Monopolistic Competition in the Qual- ity Space}

In this section, we analyze how firms compete monopolistically in the quality space for given locations. We adopt a setup in the spirit of Mussa and Rosen (1978), Gabszewicz and Thisse (1979), Bresnahan (1980) and Shaked and Sutton (1982), in which consumers value quality at a linear rate. We assume that the marginal cost of production increases sufficiently with quality so that in equilibrium, lower-valuation consumers prefer to buy goods other than those of the current technological leader. Within this framework, we 
analyze the static determinants of prices and profits for a given quality spacing.

\subsection{Preferences}

There are two goods: a homogeneous good $D$ and a good $Q$ that is differentiated in quality and supplied at different quality levels indexed by $n\left(q_{n}\right)$. Individuals derive utility from consumption of the two goods. Their utility is linear in the quantity of the homogeneous good. Each individual consumes either one or zero units of the differentiated good $Q$. Apart from this binary decision, the consumer can choose among different qualities $q$ of this good. The individual's utility function is defined as: ${ }^{6}$

$$
u_{v}(q, d)=v \cdot q+d
$$

where $v$ is a parameter that determines the desire to consume quality (and the willingness to pay for it). In the following, we will therefore call $v$ the valuation of quality, or simply the valuation. Note that no consumption of $\operatorname{good} Q$ is equivalent to a consumption of quality zero at price zero.

The total number of individuals equals $L$. Individuals value quality differently, i.e., they have different values of $v$. We define the resulting cumulative density function of valuations within the total population as

$$
G(v):[0, \infty) \rightarrow[0,1]
$$

which is assumed to have bounded support, i.e., $\operatorname{supp}(G)=[\underline{\mathrm{v}}, \overline{\mathrm{v}}]$, where $0 \leq \underline{\mathrm{v}}<\overline{\mathrm{v}}<\infty$.

\subsection{Production}

Good $D$ is produced competitively with constant returns to scale and labor as the only factor. Normalizing its price makes good $D$ the numéraire. The production technology of the $Q$-type goods depends on the good's level of quality. A firm that enters the $Q$-market to produce the good of quality $q \in(0, \infty)$ must acquire a blueprint at the fixed cost

$$
F(q)=\phi q^{\theta} \quad \theta>1
$$

\footnotetext{
${ }^{6}$ We take this preference structure from Mussa and Rosen (1978). This structure is also similar to the formulation of Shaked and Sutton (1982), who assume a multiplicative structure between the homogeneous and the differentiated good.
} 
labor units. We thus assume that blueprints for higher qualities are always more expensive.

Having acquired a blueprint for quality $q$, the firm can produce its quality of good $Q$ at constant marginal cost

$$
c(q)=\varphi q^{\theta}
$$

labor units. The parameters $\phi, \varphi>0$ govern production costs. We explicitly assume that the fixed cost of entry and the marginal cost are proportional to one another (the parameter $\theta$ is the same for both) and that both are increasing and convex in quality.

\subsection{Optimal Pricing}

Our aim is to characterize an equilibrium in which firms enter the industry at the optimal quality level and subsequently engage in monopolistic pricing. The equilibrium is solved using backward induction: we first determine the prices at given quality levels and subsequently analyze entry decisions.

We begin by characterizing the general pricing solution for an arbitrary distribution of a countable set of qualities. ${ }^{7}$ The price of quality $q_{i}$ is denoted by $p\left(q_{i}\right)$. For notational simplicity, we set $p_{n}=p\left(q_{n}\right)$ and $c_{n}=c\left(q_{n}\right)$, where $q_{n}$ is the quality level produced by firm $n$. We index firms by $n \in\{0,-1,-2, \ldots,-N\}$ and order firms by their quality level so that firm 0 produces the highest quality level $q_{0}$, and all other quality levels satisfy $q_{n-1}<q_{n}$.

Firms compete in prices, i.e., each firm sets the price for its quality to maximize its operating profits, while taking other firms' prices as given. Under preferences (1), a consumer with valuation $v$ is indifferent between two goods $q_{n}$ and $q_{n+1}$ if and only if their prices $p_{n}$ and $p_{n+1}$ are such that $v q_{n+1}-p_{n+1}=v q_{n}-p_{n}$ holds. Thus, given $G(v)$ from (2) and given the prices $\left\{p_{n}\right\}_{n \leq 0}$, the $n^{\text {th }}$ firm sells to all consumers with valuations $v$ in the interval $\left[v_{n}, v_{n+1}\right]$, where

$$
v_{n}=\left\{\begin{array}{ll}
\overline{\mathrm{v}} & \text { if } n=1 \\
\frac{p_{n}-p_{n-1}}{q_{n}-q_{n-1}} & \text { if } n<1 \\
\underline{\mathrm{v}} & \text { if } n=-N
\end{array},\right.
$$

with $N \geq 0$ being the (potentially infinite) number of active firms. Firm $n$ 's market share is thus $\left[v_{n}, v_{n+1}\right]$, and the market is partitioned as shown in Figure 1: higher-valuation

\footnotetext{
${ }^{7}$ By assuming that set of firms is countable, we anticipate that, in the equilibrium of the subsequent entry game, firms must recoup their setup costs with monopoly rents. Under Bertrand competition and positive setup cost, this assumption implies that firms must be located at positive distances from one another. Thus, the number of firms is necessarily countable.
} 
consumers tend to buy from high-quality producers. Each firm (except for the top and bottom quality producers) has two direct competitors and sells to a range of consumers who value quality sufficiently to buy from this firm rather than from its lower quality direct competitor, but they do not value quality highly enough to buy from its higher-quality direct competitor.

Figure 1: Segmentation of the consumer/valuation space by quality levels.

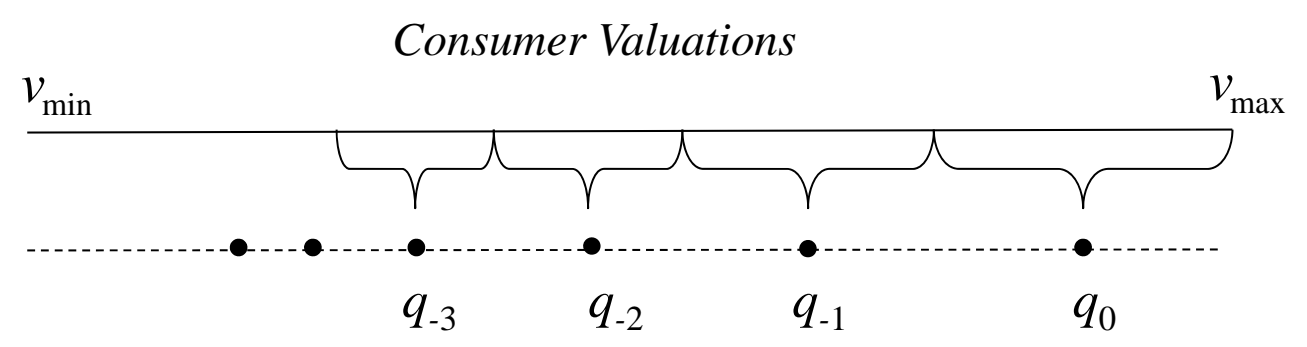

Firm Quality

Because each consumer with valuation $v \in\left[v_{n}, v_{n+1}\right]$ demands one unit of the variety produced by firm $n$, firm $n$ sells $G\left(v_{n+1}\right)-G\left(v_{n}\right)$ units of its good and solves the maximization problem:

$$
\max _{p_{n}}\left(p_{n}-c_{n}\right)\left[G\left(v_{n+1}\right)-G\left(v_{n}\right)\right] L \quad \text { s.t. (5) }
$$

The optimality condition of this problem is

$$
G\left(v_{n+1}\right)-G\left(v_{n}\right)-\left(p_{n}-c_{n}\right)\left[\frac{G^{\prime}\left(v_{n+1}\right)}{q_{n+1}-q_{n}}+\frac{G^{\prime}\left(v_{n}\right)}{q_{n}-q_{n-1}}\right]=0
$$

where expressions (5) apply. At $\underline{\mathrm{v}}$ and $\overline{\mathrm{v}}$ (the constant limits of the distribution of valuations), the derivatives in $(7)$ are defined to be zero $\left(G^{\prime}(\underline{\mathrm{v}}) \equiv 0 ; G^{\prime}(\overline{\mathrm{v}}) \equiv 0\right)$.

Previous work has established the existence and uniqueness of the pricing game for a given set of qualities under uniform distributions $G{ }^{8}$ To avoid complications arising

\footnotetext{
${ }^{8}$ Our model is a special case of the setup treated in Caplin and Nalebuff (1991). We also note that Bresnahan (1980) proves existence and uniqueness in a related setup. For the interested reader, the working paper version of this paper provides a proof of uniqueness for our specific setup.
} 
from non-existence or multiplicity of equilibria, we will therefore concentrate on the case of uniformly distributed valuations in our below analysis of the entry game. Nevertheless, we do formulate some of the following intermediate results for the general case of arbitrary distributions $G$.

As emphasized in Bresnahan (1980, 1987), existence and uniqueness constitute strong results when compared to corresponding findings in setups with a horizontally differentiated market à la Hotelling. Indeed, D'Aspremont et al. (1979) show that the pricing stage of Hotelling's location game is plagued by the non-existence of equilibria in pure strategies. ${ }^{9}$ To the contrary, vertically differentiated markets sustain a pure strategy pricing equilibrium under uniform distributions of valuations.

With (6) and (7), the operating profits can be written as

$$
\pi_{n}=\left(p_{n}-c_{n}\right)^{2}\left[\frac{G^{\prime}\left(v_{n+1}\right)}{q_{n+1}-q_{n}}+\frac{G^{\prime}\left(v_{n}\right)}{q_{n}-q_{n-1}}\right] L
$$

These operating profits, in turn, exhibit the following important regularity.

Lemma 1 Let $\left\{q_{n}\right\}_{n \leq 0}$, (4), (5) and (7) define a system with equilibrium prices $\left\{p_{n}\right\}_{n \leq 0}$ and profits $\left\{\pi_{n}\right\}_{n \leq 0}$. For a given $\chi>0$, consider the transformed system defined by $q_{n}^{\prime}=$ $\chi q_{n}, \varphi_{n}^{\prime}=\chi^{1-\theta} \varphi_{n},(4),(5)$ and (7). This transformed system has the solution $\left\{p_{n}^{\prime}\right\}_{n \leq 0}$, and the corresponding $\left\{p_{n}^{\prime}\right\}_{n \leq 0}$ and $\left\{\pi_{n}^{\prime}\right\}_{n \leq 0}$ satisfy

$$
p_{n}^{\prime}=\chi p_{n} \quad \text { and } \quad \pi_{n}^{\prime}=\chi \pi_{n} \quad \forall n .
$$

Proof. The thus-defined prices $p_{n}^{\prime}$ solve the transformed optimality conditions (7). The relation for $\pi_{n}^{\prime}$ follows from (4), (5) and (8).

The lemma states that if all quality levels and marginal productivities are scaled in suitable proportions, and thus affect marginal production costs according to (4), then equilibrium prices and profits constitute a constant proportion of the marginal production costs. This specific scaling property will be important for the entry game, leading to a particular regularity in the endogenous location pattern, as shown below.

\footnotetext{
${ }^{9}$ The authors write that "no [pure-strategy] equilibrium price solution will exist when both sellers are not far enough from each other." This specific result derives from a discontinuity in profits that arises when firms undercut the adjacent competitor and take over its entire market. As a result, profits are not quasi-concave and standard theorems for existence and uniqueness do not apply. It is worth emphasizing that in this classical model, this pathological outcome also affects the model under a uniform distribution of consumers.
} 
While Lemma 4 applies to general distributions $G$, we will restrict the setup to the case of uniformly distributed valuations for the remainder of the paper. Formally, the distribution $G$ is

$$
G(v)=\left\{\begin{array}{cl}
0 & \text { if } v<\underline{\mathrm{v}} \\
(v-\underline{\mathrm{v}}) /(\overline{\mathrm{v}}-\underline{\mathrm{v}}) & \text { if } v \in[\underline{\mathrm{v}}, \overline{\mathrm{v}}] . \\
1 & \text { if } v>\overline{\mathrm{v}}
\end{array} .\right.
$$

which guarantees the existence of a unique price equilibrium.

Before turning to the actual entry game, we below take a moment to look at the equilibrium prices under uniformly distributed valuations and a specific, regular spacing of qualities $q_{n}$.

\subsection{Pricing with Equal Relative Spacing}

In this subsection, we solve the equilibrium prices under two restrictions. The first condition is that the minimum consumer valuation is zero $(\overline{\mathrm{v}}=0) \cdot{ }^{10}$ Our second condition is that qualities exhibit a particularly regular pattern, which we will call the equal relative spacing property.

$$
\gamma q_{n-1}=q_{n} \quad \forall n
$$

Condition (10) may seem arbitrary at first but will later prove to be important. It implies that each quality is a constant fraction higher than the immediately preceding quality. It implies that each quality is a constant fraction higher than the immediately preceding one. Our focus on this special case is motivated in the subsequent section 3, which establishes how endogenous firm entry generates exactly the entry pattern that is assumed in (10).

In the case of uniformly distributed valuations and equal relative spacing, the system (7) becomes

$$
p_{n}= \begin{cases}\frac{1}{2}\left[c_{0}+\left(1-\frac{1}{\gamma}\right) q_{0} \bar{v}+p_{-1}\right] & \text { if } n=0 \\ \frac{1}{2}\left[c_{n}+\frac{1}{\gamma+1} p_{n+1}+\frac{\gamma}{\gamma+1} p_{n-1}\right] & \text { if } n<0\end{cases}
$$

In this case, the equilibrium prices of firms can be solved explicitly, and we formulate the following lemma.

\footnotetext{
${ }^{10}$ This condition mainly saves notation; in the Online Appendix, we compute equilibrium prices and provide comparative statics for the case of uniform distributions on $[\underline{\mathrm{v}}, \overline{\mathrm{v}}]$ with $\underline{\mathrm{v}}>0$.
} 
Lemma 2 Assume that the cost structure (4) and equal relative spacing in quality, i.e. (10), hold and $\bar{v}=0$. Then prices are

$$
p_{n}=\lambda^{n} A+\alpha c_{n} \quad \forall \infty \leq n \leq 0
$$

where

$$
\begin{aligned}
\alpha & =\frac{\gamma+1}{2(\gamma+1)-\gamma^{\theta}-\gamma^{1-\theta}} \\
\lambda & =\gamma+1+\sqrt{\gamma^{2}+\gamma+1} \\
A & =\frac{\lambda}{2 \lambda-1}\left(\left[1-\alpha\left(2-\gamma^{-\theta}\right)\right] c_{0}+\frac{\gamma-1}{\gamma} q_{0} \overline{\mathrm{v}}\right) .
\end{aligned}
$$

Proof. Substituting $u_{n}=p_{n}-\alpha c_{n}$ into (11) yields the second-order recursive series

$$
2\left[u_{n}+\alpha c_{n}\right]=c_{n}+\frac{1}{\gamma+1}\left[u_{n+1}+\alpha c_{n+1}\right]+\frac{\gamma}{\gamma+1}\left[u_{n-1}+\alpha c_{n-1}\right]
$$

for $n>1$. With $\alpha$ from (13), equation (16) becomes $2(\gamma+1) u_{n}=u_{n+1}+\gamma u_{n-1}$. Now notice that the type of equation

$$
X^{2}-2(\gamma+1) X+\gamma=0
$$

has two roots, $\lambda=\left[\gamma+1+\sqrt{\gamma^{2}+\gamma+1}\right]$ larger than unity and $\mu=\left[\gamma+1-\sqrt{\gamma^{2}+\gamma+1}\right]$, smaller than unity. The general solution to the recursive series (16) is thus

$$
p_{n}=A \lambda^{n}+B \mu^{n}+\alpha c_{n}
$$

Since $\lim _{n \rightarrow-\infty} p_{n}$ must be finite and non-negative, $B=0$. Equation (18) for $n=0$ is $2 p_{0}=c_{0}+\left(q_{0}-q_{-1}\right) \overline{\mathrm{v}}+p_{-1}$ and, in combination with equation (18) for $n=-1$ implies

$$
2\left(A+\alpha c_{0}\right)=c_{0}+\left(q_{0}-q_{-1}\right) \overline{\mathrm{v}}+\left(A / \lambda+\alpha c_{-1}\right)
$$

Solving for $A$ proves the claim.

Lemma 2 shows that the equilibrium price of firm $n$ under equal relative spacing (12) features a constant markup term $\alpha$ and an auxiliary term $\lambda^{n} A$. This auxiliary term may be of either sign. This term derives from the fact that the first-order condition for firm 0 is different from the first-order condition of all other firms because firm 0 faces only one 
competitor instead of two $\left(G^{\prime}\left(v_{0+1}\right)=0\right.$ in $\left.(7)\right)$. The sign and size of $A$ depends on the relative scarcity of quality compared to valuations. In particular, if $\overline{\mathrm{v}}$ is relatively small and close to $v_{0}$, firm 0 sells its top quality only to a small set of consumers, which implies that its demand elasticity is relatively large, since small price decreases induce losses of large fractions of its market. Consequently, the markup of the top quality firm is relatively small, which is reflected $A$ being small and potentially negative. Conversely, if $\overline{\mathrm{v}}$ is very large, then the top firm serves a large market segment, faces a low demand elasticity and charges high markups. In this case, the corresponding value $A$ in (15) is positive and large.

Of course, for equation (12) to characterize equilibrium prices, the relative markups $p_{n} / c_{n}-1=\left(\lambda / \gamma^{\theta}\right)^{n} A+\alpha-1$ must be positive, which is indeed the case, as the following argument shows. For parameter values that imply $A<1-\alpha$, the firm producing the top quality cannot operate profitably and thus exits the market. This implies that (after shifting indices) the quality of the active top firm, $q_{0}$, drops such that, by (4) and (15), $A$ increases. Thus, all top firms exit the market until $A \geq 1-\alpha$ holds. It also holds that $A \geq 1-\alpha$ is a sufficient condition for markups of all firms to be positive. The formal proof of this statement is relegated to the Appendix.

Lemma 2 has shown that the price schedule (12) decomposes into two components - a constant markup over production costs and an auxiliary term stemming from the border condition. Taken together, they constitute an example of variable markups - variable in the dimension of quality and depending on the model parameters. The comparative statics of prices are therefore non-trivial, and we formulate them in the following lemma.

Lemma 3 Assume that (4) and (10) hold and $\bar{v}=0$. Then,

(i) prices $p_{n}$, markups $p_{n} / c_{n}$ and the slope of the pricing schedule $\left(p_{n+1}-p_{n}\right)$ are increasing in $\bar{v}$ for all $n$.

(ii) prices $p_{n}$ are increasing in $\varphi$ and in $q_{0}$ for all $n$.

(iii) markups $p_{n} / c_{n}$ are decreasing in $\varphi$ for all $n$.

Proof. See Appendix.

Part (i) of the lemma describes the effects of an upward shift of the range of valuations, which shifts demand toward the top qualities. Under this shift, the firm producing the top quality serves a larger market segment, which translates into a less elastic demand and results in an increased markup. This price increase of the top-quality firm increases all 
prices $\left(p_{n}\right)$ and all relative markups $\left(p_{n} / c_{n}\right)$ in the market, but the upward shift in valuations benefits high-quality firms relatively more, such that the pricing schedule steepens (all $p_{n+1}-p_{n}$ increase)..$^{11}$

Part (ii) describes the effect of an increase in $q_{0}$ at constant $\gamma$, which is equivalent to an equal percentage increase of all qualities supplied. Just as in the case of upward-shifting demand, firms react to this shift in the set of qualities with price increases. However, the nature of the shift in $q_{0}$ on prices is different in this case: the increases in prices simply reflect the increases in production costs that firms must pass on to consumers. A similar effect materializes when production costs increase proportionally. In this case, prices increase without a quality upgrade .

Part (iii) of Lemma 3 shows how marginal productivity impacts relative markups. Not surprisingly, a uniform increase in the marginal production costs of all firms increases the prices of all firms. This increase in prices induces consumers to substitute towards lower qualities. Thus, the market share of the top-quality firm shrinks and its market power falls, inducing the top firm to charge lower markups. This increase in competition at the top end of qualities, in turn, propagates downward and lowers all prices.

We highlight that these comparative statics are very different from those in horizontally differentiated markets. Thus, in the classical Hotelling model (for a given supply of varieties), a uniform change in the cost parameter leaves markups unaltered because it does not affect the relative trade-off between varieties. The key reason for the differences in the impact of the parameters on equilibrium prices is that in our setup with a vertically differentiated market, the border condition of the top firm - and thus all relative prices is affected. ${ }^{12}$ Concurrently, we note that, parallel to Hotelling's setup, the overall market size $L$ does not impact firms' prices (or, hence, their market segments), since the recursive pricing formula (11) and, indeed, the generic optimality condition (7) are independent of $L$.

Of course, the price changes described in Lemma 3 have an impact on market shares,

\footnotetext{
${ }^{11}$ In the appendix, we document a similar comparative static for the lower bound of the distribution of the valuations: increasing the lowest valuation decreases all prices and decreases the slope of the pricing schedule. We empirically examine the importance of the support of income distributions on markups in a model of how quality is priced to market in Auer et al. (2014).

${ }^{12}$ By contrast, in Vogel (2008) transportation costs do impact markups for a given number of entrants due to their effect on the equilibrium spacing: specifically, higher transportation costs mitigate the importance of heterogeneity in productivity and thus the equilibrium degree of isolation between firms.
} 
i.e., the cutoff valuations. These are formulated in the following corollary.

Corollary 1 Assume that (4) and (10) hold and $\bar{v}=0$. Then, each of the equilibrium cutoff-valuations $v_{n}$ is increasing in $\bar{v}$.

Proof. This follows directly from (5) and Lemma 3 (i).

Corollary 1 shows that an exogenous expansion of demand for top qualities through the 'arrival' of consumers with a high valuation for quality ( $\overline{\mathrm{v}}$ increases) crowds down other consumers: the arrival of high-valuation consumers increases all prices but the prices of high-quality firms increase the most. Consequently, cutoffs move up, i.e., the pre-existing set of consumers (weakly) downgrade their quality choices.

Finally, we can assess the impact of entry of an additional firm on the markups of existing ones and on consumers' equilibrium quality choice. The pricing rule allows us to make an intuitive and simple statement regarding the effect of entry on the markups of existing firms.

Lemma 4 Assume that (4) and (10) hold and $\bar{v}=0$. The entry of an additional firm at the top end of the quality spectrum at $q_{1}=\gamma q_{0}$

(i) weakly decreases the markup of each established firm,

(ii) weakly flattens the pricing schedule $\left(p_{n+1}-p_{n}\right)$, and

(iii) weakly decreases cutoff valuations $v_{n}$.

Proof. See Appendix.

This lemma shows that entry of a new technological leader increases competition and lowers prices. For given a $\bar{v}$, the new technological leader faces a smaller market segment and therefore a higher demand elasticity than the previous technological leader did before entry. ${ }^{13}$

\footnotetext{
${ }^{13}$ Technically, entry at the top reduces the constant from $A$ to $\widetilde{A}$. The incumbent technological leader becomes firm -1 and is thus "isolated" from the new constant. If the constant is negative, this isolation might be considered beneficial for the firm. However, Lemma 4 documents that the total effect is always such that prices (and thus markups) of pre-existing firms weakly decrease once entry occurs. In addition, note that the Lemma covers two cases. First, the additional firm engages in production and affects the whole market by depressing markups. Second, in the case of preemptive entry, it does not pay for the additional firm to produce and sell its goods, and consequently, it leaves the market unaffected. Since this second case may indeed occur, the entry of additional firms decreases the markup of any preexisting firm only weakly.
} 
Lemma 4 also establishes that entry weakly flattens the pricing schedule $\left(p_{n+1}-p_{n}\right)$ and decreases the cutoff valuations $v_{n}$. By lowering the consumer prices of all goods, but in particular those of high-quality goods, the entry of a new technological leader (weakly) increases the equilibrium cutoff valuations $\mathrm{v}_{n}$ for all consumers.

This section has established comparative statics of prices for given entry and analyzed the impact of entry by an additional firm on prices. In all cases, we have considered the special case of equal relative spacing. We did not, of course, choose the special case of equal relative spacing by accident: endogenous firm entry generates exactly the pattern described in (10). We now turn to the entry game and the resulting endogenous quality spacing.

\section{Endogenous Spacing with Exogenous Growth}

This section analyzes firms' endogenous choice of qualities. The general equilibrium concept is fairly standard: we assume that there is an unbounded pool of ex-ante identical entrants that face deterministic entry costs (3). A firm enters if and only if the net present value of future operating profits covers these entry costs. The future profits accrue under the rationally anticipated unique price equilibria. ${ }^{14}$ Firms make an endogenous quality choice when entering, which pins down the firm's quality permanently. Firms choose their quality, taking the qualities of all established firms as given but endogenizing the impact of their choice on the timing and quality choice of all future entrants.

The less-standard feature of our setup is the introduction of a dynamic dimension. Specifically, we assume that time is continuous and that productivity in the $Q$-sector grows at the constant rate $a$, which is exogenously given. Indexing the cost parameters $\varphi$ and $\phi$ with time subscripts, we have

$$
\varphi_{t}=e^{-a t} \varphi \quad \text { and } \quad \phi_{t}=e^{-a t} \phi
$$

Our main contribution in this paper is showing that in this dynamic setup, free entry supports equilibria with equal relative spacing of firms, endogenously generating quality levels that satisfy (10). In other words, our analysis focuses on a periodic, quasi-stationary

\footnotetext{
${ }^{14}$ Recall that we assume that valuations are distributed uniformly on the interval $[\underline{\mathrm{v}}, \overline{\mathrm{v}}]$ according to (9). We also note that price equilibria do not need to be unique for firms to compute expected profits and entry decisions. In fact, the results of the current section may be be generalized when conveniently defining expectations about future price equilibria. However, we focus on uniform distributions of valuations for the sake of simplicity and to avoid unnecessary discussions regarding these expectations.
} 
equilibrium in which each firm enters the industry as the technological leader and successively transits through the product cycle as it becomes superseded by subsequent innovators.

The key advantage of studying the entry game in such a dynamic setting is that this choice makes the inherently asymmetric entry problem - with a top quality end as an upper bound - effectively symmetric. Specifically, we first analyze the entry problem separately for a single firm and then show that under the adequate initial conditions, the new entrant's quality satisfies the regular pattern of equal relative spacing (10). In the second step, we observe that the optimization problems of all consecutive entrants are identical up to a scaling factor and hence replicate (10). Thus, the series of problems is self-similar, or effectively symmetric. ${ }^{15}$ The infinite sequential entry problem thus collapses to one of a single firm, avoiding problems that typically arise in simultaneous-entry games with an asymmetric setup (e.g., as in Vogel (2008)). ${ }^{16}$

We begin the technical part of the analysis with the situation described in the previous section: the set of active firms is $\{0,-1,-2, \ldots\}$, and the firms are ranked in ascending order by quality so that a higher firm index corresponds to a firm producing higher quality $q \in\left\{q_{n}\right\}_{n \leq 0}$. Moreover, all established qualities $q_{n}$ are assumed to satisfy (10).

We assume that a plant established to produce quality $q_{m}$ automatically holds the blueprints for all qualities between $q_{m-1}$ and $q_{m}$, where $q_{m-1}$ is the next-lowest quality level. This assumption restricts the entry of additional firms to quality levels above the pre-existing ones $\left(q_{m+1} \geq q_{m}\right){ }^{17}$

Firms that gradually establish themselves at the top end of the quality spectrum are indexed by $m \geq 1$. Let $t_{m}$ be the entry date of the $m^{\text {th }}$ additional firm (implying $0 \leq$ $\left.t_{1} \leq t_{2} \leq ..\right)$. Further, let $q_{m}$ denote its quality level $\left(q_{0} \leq q_{1} \leq q_{2} \leq ..\right)$. It will prove

\footnotetext{
${ }^{15}$ This strong property relies on the assumptions that we make on production costs (3) and (4) and, importantly, on the fact that both fall at the same rate $a$, as specified in (19).

${ }^{16}$ In fact, the resulting complications would be substantial in our setup, because the clear ranking of the quality line prevents us from using the symmetry properties that arise in models such as Salop (1979), who assumes that the space is represented by a circle. In a quality setup, any attempt to "close the circle" fails, since it would amount to identifying the highest-quality good with the lowest-quality good.

${ }^{17}$ We note that this is a strong assumption. In an unrestricted entry game, we cannot exclude a priori that an entrant would choose a quality below that of the technological leader. However, we focus on the case of top quality entry only, since in international patent law, entrants are granted a patent only if an innovation contains an element of novelty that improves upon existing technology. Patents thereby also grant current technology leaders protection from future entry by lower-quality competitors (see World Intellectual Property Organization (2004)). Chor and Lai (2013) label this the "inventive step requirement" and theoretically examine its welfare-maximizing level in a model of Schumpeterian growth following Aghion and Howitt (1992).
} 
convenient to express the quality choice of the $m^{t h}$ entrant relative to the highest quality of all incumbents $\left(q_{m-1}\right)$ as

$$
\gamma_{m}=q_{m} / q_{m-1} \quad m \geq 1
$$

At time $\tau \in\left[t_{m+k}, t_{m+k+1}\right)$, the set of quality levels available to the market is $\left\{q_{n}\right\}_{n \leq m+k}$. Current prices are determined by equation (7) and depend on all currently produced quality levels and on productivities evolving according to equation (19). Consequently, at time $\tau \in\left[t_{m+k}, t_{m+k+1}\right)$, the operating profit of the $m^{t h}$ additional firm, given by equation (8), is a function of the qualities $\left\{q_{n}\right\}_{n \leq m+k}$ and time $\tau$. Note that for a given set of competitors, profits depend on time only through productivity $\varphi e^{-a t}$ and we thus conveniently express the operating profits of firm $m$ at time $\tau$ as $^{18}$

$$
\pi_{m}\left(\varphi e^{-a \tau}, q_{m+k}, \gamma_{m+k}, \gamma_{m+k-1}, \gamma_{m+k-2}, \ldots, \gamma_{1}, \gamma\right) \quad \tau \in\left[t_{m+k}, t_{m+k+1}\right)
$$

When defining the product

$$
\Gamma_{m, k}=\prod_{j=1}^{k} \gamma_{m+j}
$$

we have $q_{m+k}=\Gamma_{m, k} q_{m}$ so that at time $t_{m}$, the present value of the flow of operating profits for a potential entrant is equal to

$$
\Pi\left(\gamma_{m}, t_{m}\right)=\sum_{k \geq 0} \int_{t_{m+k}}^{t_{m+k+1}} e^{-r\left(\tau-t_{m}\right)} \pi_{m}\left(\varphi e^{-a \tau}, \Gamma_{m, k} \gamma_{m} \Gamma_{0, m-1} q_{0}, \gamma_{m+k}, \gamma_{m+k-1}, \ldots, \gamma_{1}, \gamma\right) d \tau
$$

where the parameter $r$ is the constant rate at which firms discount future profits.

With these definitions, we can formulate the entry decision. The $m^{\text {th }}$ firm chooses its entry date $\left(t_{m}\right)$ and its location on the quality line $\left(\gamma_{m}\right)$. With the choice of $\gamma_{m}$, the firm maximizes the present value of profits at time $t_{m}(21)$ net of its entry costs (3). Given the existing spacing $\gamma_{m-1}, \gamma_{m-2}, \ldots, \gamma_{1}, \gamma$, and conditional on the entry date $t_{m}$, the $m^{\text {th }}$ optimal quality choice is

$$
\begin{gathered}
\hat{\gamma}_{m}\left(\gamma_{m-1}, \ldots, \gamma_{1}, \gamma\right)=\underset{\tilde{\gamma} \geq 1}{\arg \max }\left\{\sum _ { k \geq 0 } \int _ { \tilde { t } _ { m + k } } ^ { \tilde { t } _ { m + k + 1 } } e ^ { - r ( \tau - t _ { m } ) } \pi _ { m } \left(\varphi e^{-a t}, \tilde{\Gamma}_{m, k} \tilde{\gamma} \Gamma_{0, m-1} q_{0}, \hat{\gamma}_{n+k}, \ldots\right.\right. \\
\left.\left.\ldots \hat{\gamma}_{n+k-1}, \hat{\gamma}_{n+1}, \tilde{\gamma}, \gamma_{n-1}, \ldots, \gamma_{1}, \gamma\right) d \tau-\phi e^{-a t_{m}}\left(\tilde{\gamma} \Gamma_{0, m-1} q_{0}\right)^{\theta}\right\}
\end{gathered}
$$

\footnotetext{
${ }^{18}$ Note that prices and profits $\pi_{m}$ are unique under assumption (9).
} 
Here, $\tilde{\Gamma}_{m, k}$ denotes, in parallel to $(20)$, the product of the $k$ future optimal relative spacing parameters, given that the $m^{t h}$-entrant plays $\tilde{\gamma}$ :

$$
\tilde{\Gamma}_{m, k}=\prod_{j=1}^{k} \hat{\gamma}_{m+j}\left(\hat{\gamma}_{m+j-1}, \hat{\gamma}_{m+j-2}, \ldots \tilde{\gamma}_{m}, \gamma_{m-1}, . ., \gamma_{1}, \gamma\right)
$$

We stress that all future entry dates $\tilde{t}_{m+j}$ and location choices $\hat{\gamma}_{m+j}(j \geq 1)$, and hence $\tilde{\Gamma}_{m, j}$, are functions of firm $m$ 's choice $\tilde{\gamma}$. This dependence is indicated by the tilde " $\sim$. For expositional purposes, however, the arguments $\hat{\gamma}_{m+j}(\tilde{\gamma}), \tilde{\Gamma}_{m, j}(\tilde{\gamma}), \tilde{t}_{m+j}(\tilde{\gamma})$ are suppressed in (22) and in the equations further below. The $m^{\text {th }}$ firm's entry date is determined by the free entry condition, i.e., the requirement $\Pi\left(\gamma_{m}, t_{m}\right) \geq F\left(\gamma_{m}, q_{m-1}\right)$. Formally, we write

$$
\begin{array}{r}
t_{m}=\inf \left\{t \geq t_{m-1} \mid \sup _{\tilde{\gamma} \geq 1}\left[\sum _ { k \geq 0 } \int _ { \tilde { t } _ { m + k } } ^ { \tilde { t } _ { m + k + 1 } } e ^ { - r ( \tau - t _ { m } ) } \pi _ { m } \left(\varphi e^{-a t}, \tilde{\Gamma}_{m, k} \tilde{\gamma} \Gamma_{0, m-1}^{*} q_{0}, \hat{\gamma}_{m+k}, \hat{\gamma}_{m+k-1}, \ldots\right.\right.\right. \\
\left.\left.\left.\ldots, \hat{\gamma}_{m+1}, \tilde{\gamma}, \gamma_{m-1}^{*}, \gamma_{m-2}^{*} \ldots, \gamma_{1}^{*}, \gamma\right) d \tau-\phi e^{-a t_{m}}\left(\tilde{\gamma} \Gamma_{0, m-1} q_{0}\right)^{\theta}\right] \geq 0\right\}
\end{array}
$$

where the asterisk * denotes the equilibrium locations:

$$
\gamma_{1}^{*}=\hat{\gamma}_{1}(\gamma) \quad \text { and } \quad \gamma_{k}^{*}=\hat{\gamma}_{k}\left(\gamma_{k-1}^{*}, \gamma_{k-2}^{*}, \ldots, \gamma_{1}^{*}, \gamma\right)
$$

and $\Gamma_{0, k}^{*}$ is defined in parallel to $(20)$ as the product of the equilibrium $\gamma_{j}^{*}$

$$
\Gamma_{0, k}^{*}=\prod_{j=1}^{k} \gamma_{j}^{*}
$$

Optimal quality choices (22) and the free entry conditions (23) for all $m \geq 1$ determine the equilibrium of the entry game. The first important result of this section concerns the solution for the system (22) - (23) and is formulated in the following proposition.

Proposition 1 Let $(\theta, \phi, \varphi, L, r, a)$ be a combination of positive parameters. Then, (i) for a given $q_{m}$ and $\left\{\gamma_{n}\right\}_{n \leq m}$, the entry date $t_{m+1}^{*}$ and the optimal location $\gamma_{m+1}^{*}$ are well-defined and unique. The choice $\gamma_{m+1}^{*}$ does not depend on $q_{m}$.

(ii) there is a $\bar{\gamma}(\theta, \phi, \varphi, L, r, a)>1$ such that, if $\gamma_{n}=\bar{\gamma}$ for all $n \leq m$, then

$$
\gamma_{m+1}^{*}=\bar{\gamma}
$$

holds. In this case, time intervals between consecutive entries are constant:

$$
t_{m+1}^{*}-t_{m}^{*}=\Delta=\ln (\bar{\gamma})(\theta-1) / a
$$




\section{Proof. See Appendix.}

Part (i) of the proposition states that there is a well-defined, unique equilibrium with finite relative spacing $\gamma_{m}=q_{m} / q_{m-1} \in[1, \infty)$. Notably, the relative spacing of the $m+1^{\text {th }}$ firm does not depend on the level of previous qualities, $\left\{q_{n}\right\}_{n \leq m}$, but only on their relative spacing $\left\{\gamma_{n}\right\}_{n \leq m}$. This result is a reflection of Lemma 1 and is ultimately a result of the model's scaling property. A new entrant $m+1$ compensates a proportional increase in existing qualities $q_{n}(n \leq m)$ by postponing the entry date, which lowers fixed entry costs and marginal costs. The entry date is delayed, but location decisions are unaffected.

Part (ii) of Proposition 1 shows that a specific type of equilibria can arise that exhibit a particularly convenient and regular spacing pattern: equal relative spacing $\gamma_{n}=\bar{\gamma}$ applies to the qualities of all incumbents as well as those of all future entrants. Note, however, that part (ii) of the proposition does not involve uniqueness. In particular, the uniqueness of the equilibrium from part (i) does not necessarily imply the uniqueness of the equilibrium in part (ii). ${ }^{19}$

The lack of uniqueness of the equilibrium from part (ii) should not come as a surprise and is well-known to arise in some models with strategic complementarity in entry. Many comparable models of free entry to markets are subject to the same indeterminacy (see Capozza and Van Order (1980) and, more recently, Vogel (2008)). ${ }^{20}$ In our current modeling setup, a strategic complementarity would mean that a dense spacing of the existing qualities would make it more attractive for new entrants to choose a small $\gamma$ on their own (thus replicating the dense spacing). At first sight, such an effect may seem counterintuitive. However, considering the simultaneous choice of location and entry date, one objective of the current entrant is to delay the entry of the next entrant. One way to accomplish this is to commit to lower future prices. Such a commitment can be reached if preexisting qualities are densely spaced, since the impact of the additional entrant on the incumbent top-firm's pricing rule is lower in this case. As long as the value of commitment rises with the density of preexisting qualities relative to the loss in instantaneous profits, strategic complementarities can potentially arise, thus giving rise to the possibility of multiple equilibria. In sum,

\footnotetext{
${ }^{19}$ This potential non-uniqueness is comparable to that in growth models in which unique investment strategies - given a set of relevant state variables - do not guarantee unique steady states.

${ }^{20}$ More generally, strategic complementarities are typically involved when multiple equilibria arise (see also Cooper and John (1988)).
} 
we cannot discard strategic complementarities in the quality choice and may thus face multiplicity of equilibria with equal relative spacing in our model.

An equilibrium described in Proposition 1 (ii) that is characterized by $\gamma_{n}=\bar{\gamma}$ for all integers $n$, can be labeled an equal relative spacing equilibrium. In the following analysis we focus on a specific one and apply the following definition.

Definition ERSE. For any given set of parameters, the Equal Relative Spacing Equilibrium (ERSE) is an equal relative spacing equilibrium with the minimal spacing $\bar{\gamma}$.

Note that under a preexisting spacing parameter equal to one $(\gamma=1)$, the optimal spacing of the first entrant $\gamma^{*}(\gamma)$ from $(24)$ satisfies $\gamma^{*}(\gamma)>\gamma$ for all $\gamma \in(1, \bar{\gamma})$ (see the proof of Proposition 1). Therefore, at the minimal symmetric $\bar{\gamma}$, characterized by $\gamma^{*}(\bar{\gamma})=\bar{\gamma}$, the following inequality holds:

$$
\left.\frac{d \gamma^{*}(\gamma)}{d \gamma}\right|_{\gamma=\bar{\gamma}}<1 .
$$

We also point out that the ERSE is unique in the special case when the distribution of valuations is sufficiently compressed (i.e., the ratio $\overline{\mathrm{v}} / \underline{\mathrm{v}}$ is small). A sketch of a proof runs as follows. Consider the choice of a representative new entrant, firm 1, under any set of established qualities $\left\{q_{0}, q_{-1}, \ldots\right\}$. By Proposition 1 (i), the relative location choice of firm $1, \gamma_{1}$, is independent of $q_{0}$. In addition, at most two firms are simultaneously active for $\overline{\mathrm{v}} / \underline{\mathrm{v}}$ small enough. ${ }^{21}$ Therefore, $\gamma_{1}$ is independent of $\left\{q_{-1}, q_{-2}, \ldots\right\}$ as well. In sum, firm 1 's relative location choice $\gamma_{1}$ is independent of all previous qualities $q_{n}(n \leq 0)$ and is therefore unique. Consequently, there is only one optimal $\gamma$ for all entrants, which means that the ERSE is the only equilibrium. ${ }^{22}$

This section has derived novel results about the regularity of firm spacing (Proposition 1). In the next section, we will discuss the ERSE and provide comparative statics.

\footnotetext{
${ }^{21}$ This is a version of the finiteness property in Shaked and Sutton (1982 and 1983). Using (7) and (9), prices and profits are quickly computed for three firms $n=0,-1,-2$. For $\underline{\mathrm{v}}$ close to $\overline{\mathrm{v}}$, the firms' qualities $\left(q_{0}=\gamma^{2} q_{-2}, q_{-1}=\gamma q_{-2}\right.$ and $\left.q_{-2}\right)$ must be sufficiently close to one another for all firms to be active. In particular, $\gamma \rightarrow 1$ as $\underline{\mathrm{v}} \rightarrow \overline{\mathrm{v}}$, which implies zero profits in the limit and contradicts free entry under any $\phi>0$.

${ }^{22}$ Technically, the intertemporal links decouple and the dynamic system effectively becomes a static one: the entrant's problem of choosing the optimal $\gamma$ is independent of the model's state variables $\left(q_{0}, q_{-1}, \ldots\right)$ and is thus replicated for each new entrant.
} 


\section{Properties of the ERSE}

This section first summarizes and illustrates some of the results presented above and then derives comparative statics of the ESRE.

\subsection{Firm Dynamics in the ERSE}

The assumption of constant technological progress in (19) introduces a dynamic dimension to the model, which gives rise to the ERSE described in Proposition 1 (ii). With the help of Lemma 2 and Lemma 4, we can illustrate how the dynamics of the equilibrium play out in detail. The prices and profits of a firm producing quality $q$ evolve as depicted by the blue and green lines in Figure 2. Each continuous section represents prices and profits when no innovation occurs. Innovations occur at regular intervals; these entry dates are marked by the vertical dashed lines. These entry dates are equidistant according to Proposition 1. At each of these dates, the firm's relative markup - and thus its operating profit - drop by a discrete amount because the new competitor reduces the incumbents' market power and its market share (compare Lemma 4 (i)).

The time profile of profit flows is shaped by two opposing forces. First, for a given set of firms, the profit flow for the top-quality firm is increasing, since productivities increase over time. These productivity gains reduce the cost of the production of all goods, spurring demand for quality and thus increasing the market share of the top-quality firm. Second, however, productivity growth also implies that firms enter the market and each existing firm's market segment narrows. In particular, as time passes, the firm serves consumers with lower and lower valuations. It becomes squeezed to the bottom end of the quality distribution, serving an ever smaller segment. Therefore, the profit flow drops to zero in the limit.

In the equilibrium illustrated in Figure 2, an entrant immediately starts producing at its entry date. However, if entry is sufficiently cheap relative to the marginal productivity, the zero profit condition may also force firms to enter the industry preemptively: at the entry date, the production of the top quality is too costly, such that there is no demand for it even when sold at marginal costs. 


\section{Figure 2: Life-cycle of a firm entering at $t^{*}=0$.}

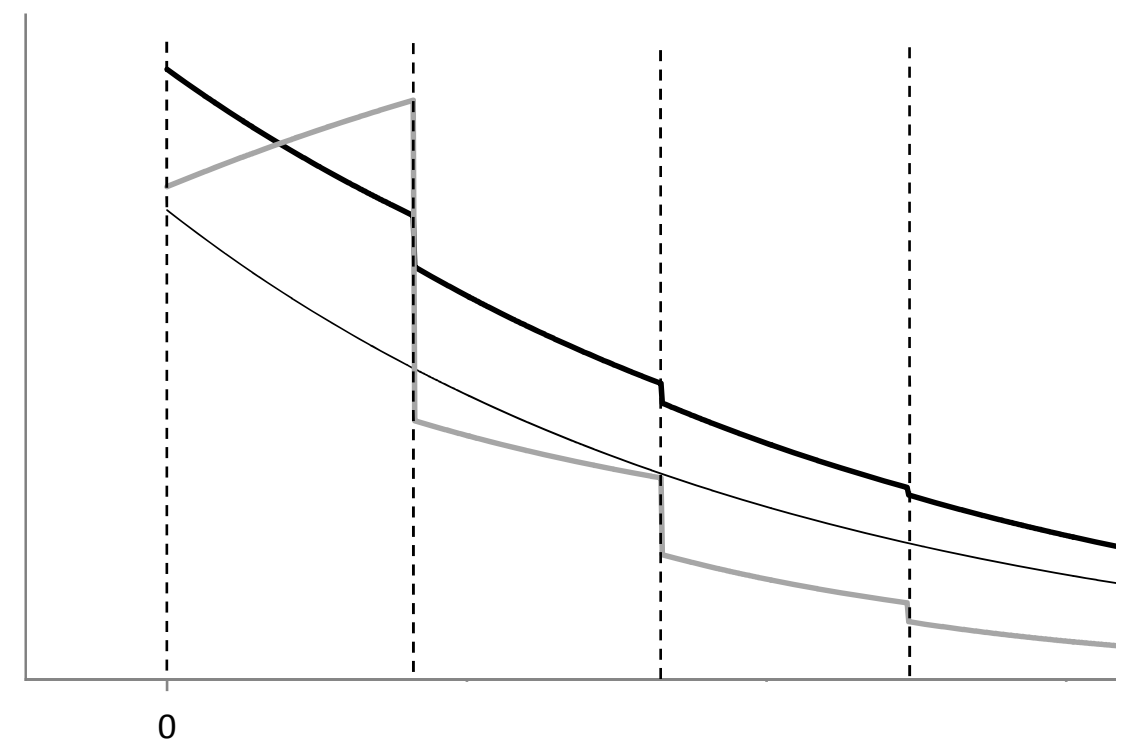

The figure depicts the evolution of marginal production costs (thin black line), prices (fat black line) and profits (fat grey line) of a firm that enters the market at $t^{*}=0$. Marginal production costs fall exogenously according to (19). The firm's prices also fall over time, largely following marginal costs. The difference between prices and marginal costs is the markup. In addition to the gradual reduction of production costs, prices drop according to Lemma 4 whenever a new firm enters the market (entry dates indicated by the dashed vertical lines to the right). At these dates, the increase in the set supplied qualities tightens competition and induces the discrete drop in prices. Profits initially increase with time, because relative markups increase and the market share of the top firm expands as production costs decrease. In the long run, a firm's market share becomes negligible and its profits approach zero. 
Figure 3 depicts such an equilibrium, where at the date of entry, a new firm's marginal cost is too high to profitably operate. In this equilibrium, entry is followed by a period during which exogenous cost reductions occur that ultimately make production of the recent entrant profitable. When productivities are just high enough to make the consumer with the maximum valuation purchase the new quality at marginal costs, the firm enters the market selling with zero markup to a negligible set of consumers. Thereafter, the profit flow of the new firm increases steeply: its set of consumers grows along with productivity. As it sells to a growing range of consumers, its demand becomes less and less elastic and its markup increases. The vertical dotted lines in Figure 2 indicate the dates when new firms start production, supplying better and better qualities. At each of these dates, the increased competition reduces markups and profits of the incumbent firm. In the long run, the firm's market share converges to zero and its profit flow approaches zero.

We finally notice that a deadweight loss arises in the case of preemptive entry. This deadweight loss is equal to the interest on entry costs accruing between the entry date and the start of production and can be eliminated by an otherwise non-distortionary tax on the entry cost $\phi$. Specifically, a tax that marginally increases entry costs postpones entry without altering the supply of qualities at any given point in time, thereby decreasing the deadweight loss without distorting firms' quality supply or prices.

To verify this statement, consider a situation with preemptive entry and a light tax that delays entry (but marginally only so that entry is still preemptive). This delay is irrelevant for supply of qualities in the period between the entry date without the tax and the entry date with the tax: in either case production of the firm's good is nil during this period. More importantly, the firm faces essentially the same optimization problem in both cases: an optimal location choice, the objective functions of which differ only by a discounting factor. More formally, assume that a firm enters the market at date $t^{*}$ but begins selling its product at $t^{* *}>t^{*}$. Its optimal quality level is $q$, so its entry cost at date $t^{*}$ is $\phi_{t^{*}} q^{\theta}$. At any time $t^{*}+\Delta t \in\left[t^{*}, t^{* *}\right]$ would the firm reconsider its quality choice? The answer to this question is obviously no: if $q$ maximizes the net present value of future profits at time $t^{*}$ (i.e., $\Pi(q)-\phi_{t^{*}} q^{\theta}$ ), then $q$ maximizes the net present value of future profits at time $t^{*}+\Delta t$ (i.e., $\left.e^{r \Delta t}\left(\Pi(q)-\phi_{t^{*}} q^{\theta}\right)\right)$ as well. This latter observation, in turn, implies that the same $q$ is optimal if the firm were to enter the market at time $t^{*}+\Delta t$ under a modified entry cost parameter $\phi^{\prime}=e^{r \Delta t} e^{a \Delta t} \phi$ - i.e. under a gross entry tax of $e^{(r+a) \Delta t}$ - since the 
modified objective function at date $t^{*}+\Delta t$ is again $e^{r \Delta t}\left(\Pi(q)-\phi_{t^{*}+\Delta t} q^{\theta}\right)$. In this case, the entering firm's total profits are zero and its quality choice and its production start are thus unchanged. ${ }^{23}$ Overall, we conclude that a tax of the gross rate $e^{(r+a)\left(t^{* *}-t^{*}\right)}$ postpones the entry date to $t^{* *}$ : in this case entry and the start of production coincide.

We also point out that the case of preemptive entry in the current setup thus parallels the one of Anderson and Engers (2001), who analyze horizontally differentiated markets in which firms can block a spot or location in a horizontally differentiated market by preemptively incurring entry costs. Just as in Anderson and Engers (2001), under preemptive entry, an entry tax postpones the excessively early entry of firms but leaves the spacing of competition unchanged, thus strictly enhancing efficiency.

\subsection{Comparative Statics of the ERSE}

We now turn to the comparative statics of the ERSE. As the first step, we exploit the model's scaling properties to establish which parameter shifts do not impact equilibrium spacing. These results are formulated in the following lemma.

Lemma 5 Let $\gamma_{1}$ be the equilibrium choice of a new entrant. Then,

(i) $\gamma_{1}$ depends on $\phi, \varphi$ and $L$ only through the ratio $\phi /(\varphi L)$, so that $\gamma_{1}(\phi /(\varphi L))$.

(ii) the transformation $(\phi, \varphi) \rightarrow \chi \cdot(\phi, \varphi)(\chi>0)$ postpones entry dates by $\ln (\chi) / a$.

(iii) $\gamma_{1}$ is constant under the transformation $\left(r^{\prime}, a^{\prime}, L^{\prime}\right) \rightarrow \chi \cdot(r, a, L)$ with $\chi>0$.

(iv) the transformation $(\underline{v}, \bar{v}) \rightarrow \chi^{-1} \cdot(\underline{v}, \bar{v})$ with $\chi>0$ preserves $\gamma_{1}$ but postpones the entry date by $\ln (\chi) / a$.

Proof. (i) Wlog, consider the first entrant, firm 1 in an ERSE. Its operating profits $\pi$ are linear in $L$, and its setup costs $F$ are linear in $\phi$. Replacing $\phi^{\prime}=\phi / L$, population $L$ factors out of the slanted brackets in (22) and the square brackets in (23). The solution $\gamma_{1}$

\footnotetext{
${ }^{23}$ The same statement is also formally derived from equation (37) in the proof of Proposition 2 in the Appendix where at entry date $t^{*}$ the operating profit and its derivative are trivially zero $\pi\left(t^{*}\right)=\pi_{\hat{\gamma}}\left(t^{*}\right)=0$ under preemptive entry. Hence, $d \bar{\gamma} / d \phi=0$ follows, i.e., the entry cost does not have an impact on the spacing parameter $\bar{\gamma}$ in this regime. For free entry (32) under (31) to hold at an unchanged distribution of qualities, any marginal change in $\phi$ must induce a change in the entry time $t^{*}$ that leaves constant the expression $\phi e^{t^{*}}$.
} 


\section{Figure 3: Life-cycle of a firm entering at $t_{0}^{*}$ under preemptive entry.}

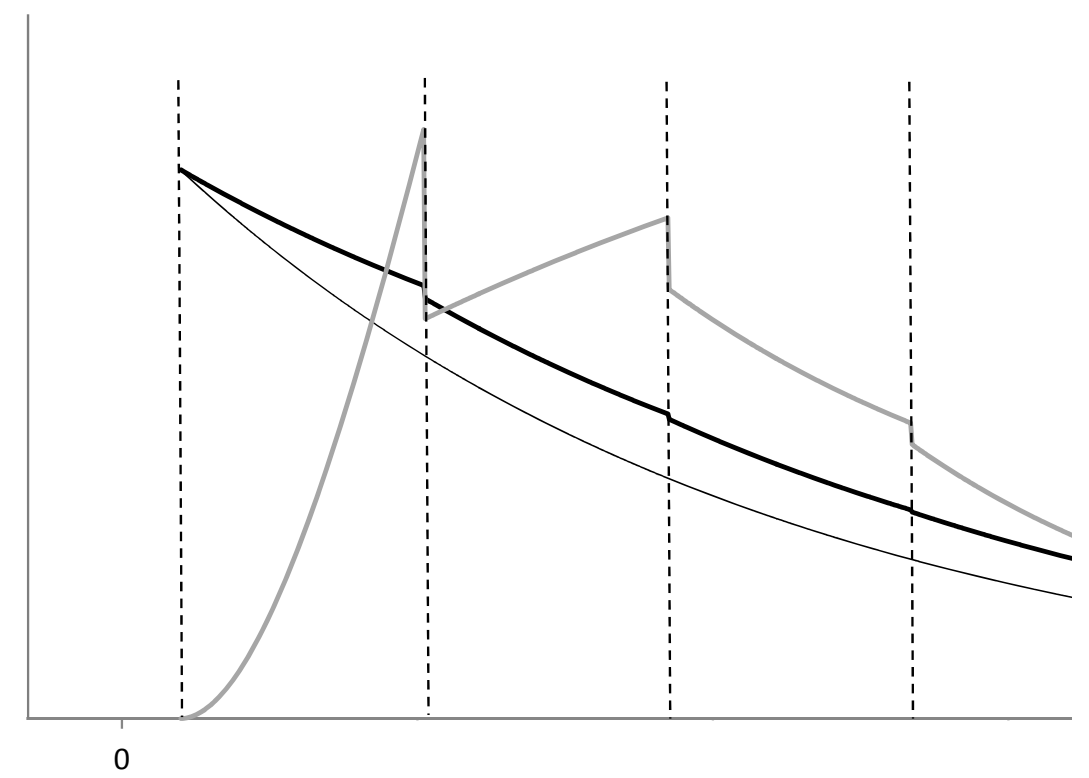

The figure depicts the evolution of marginal production costs (thin black line), prices (fat black line) and profits (fat gray line) of a firm that enters the market at $t^{*}=0$. At entry date, marginal production costs are too high so the firm does not produce for a period until its production costs have decreased sufficiently and the firm captures a non-zero market share. This happens at the date indicated by the dashed vertical line to the left: at that date, the firm becomes active in the market but serves only a negligible market share. Its markups are zero so that its marginal cost coincides with its the price. As marginal costs decrease further, its market share grows and its markup becomes positive. In parallel to the dynamics in Figure 2, the firm's markups and profits drop whenever a new entrant becomes active in the market (the dashed vertical lines to the right). In the long run, the firm's market share and profits become negligible. 
of the transformed problem is thus identical to the original one (22) - (24) and depends on $\phi^{\prime}=\phi / L$ only. This proves that $\phi$ and $L$ affect $\gamma_{1}$ only through the ratio $\phi / L$.

Next, consider any transformation of $\phi$ and $\varphi$ that preserves the ratio $\phi / \varphi$, i.e., consider $\left(\phi^{\prime}, \varphi^{\prime}\right)=\chi \cdot(\phi, \varphi)$. By $(19)$, a simultaneous transformation of time by $t^{\prime}=t+a^{-1} \ln (\chi)$ replicates the original problem and, thus, the equilibrium $\gamma_{1}$. Hence, $\phi$ and $\varphi$ affect $\gamma_{1}$ only through the ratio $\phi / \varphi$. Together, this proves claim (i).

(ii) Follows from that fact that the transformation $\left(\phi^{\prime}, \varphi^{\prime}, t^{\prime}\right)=\left(\chi \phi, \chi \varphi, t+a^{-1} \ln (\chi)\right)$ from part (i) replicates the original problem and its solution.

(iii) The transformation $\left(r^{\prime}, a^{\prime}\right)=\chi \cdot(r, a)$ is equivalent to a transformation of the time variable $t^{\prime}=\chi t$. According to the transformation formula, we have $\int_{\chi t_{o}}^{\chi t_{1}} f\left(t^{\prime}\right) d t^{\prime}=$ $\int_{t_{o}}^{t_{1}} f(\chi t) \chi d t$ for a generic function $f$. Normalizing $t_{o}=0$ and rearranging, this implies

$$
\int_{0}^{\infty} e^{-r \chi\left(\tau-t_{1}\right)} \pi_{1}(\chi t) d t=\chi^{-1} \int_{0}^{\infty} \pi_{1}\left(t^{\prime}\right) d t^{\prime}
$$

with $\pi_{1}$ as in (21). Under the simultaneous transformation $L^{\prime}=\chi \cdot L$, the expression on the right hand side is multiplied by $\chi$, since $\pi_{1}$ is linear in $L$. This implies that the integral in (21) is unchanged; the firm's objective function is thus preserved and the equilibrium $\gamma_{1}$ unchanged.

(iv) Note first, using (5) and (7), that the joint transformation $(\underline{\mathrm{v}}, \overline{\mathrm{v}}) \rightarrow \chi \cdot(\underline{\mathrm{v}}, \overline{\mathrm{v}})$ and $\left(\phi^{\prime}, \varphi^{\prime}\right)=\chi(\phi, \varphi)$ implies that optimal prices are transformed by the factor $\chi$. Hence, the net present value of firm profits $(21)$ is transformed through multiplication by $\chi$. The firm's objective function is thus unchanged except for the scaling factor $\chi$ and the equilibrium $\gamma_{1}$ is consequently unaffected. Finally, the transformation $\left(\phi^{\prime}, \varphi^{\prime}\right)=\chi(\phi, \varphi)$ is equivalent to the time shift $t^{\prime}=t+\ln (1 / \chi) / a$. This observation completes the proof.

Technically, part (i) of Lemma 5 shows that the density of spacing $\gamma_{1}$ is affected by the costs $\phi$ and $\varphi$ and market size $L$ only through the ratio $\phi /(\varphi L)$. Intuitively, since prices are independent of the market size $L$, the operating profits are proportional to $L$. Thus, an increase in the fixed costs of market entry, $\phi$, is offset by a corresponding percentage increase in the market size and leaves the equilibrium unchanged. Specifically, this joint transformation has no impact on equilibrium spacing. Similarly, an equiproportional increase in market entry cost $\phi$ and marginal production cost $\varphi$ by the factor $\chi$ does not matter for optimal spacing. In this case, however, entrants simply wait until costs have 
dropped according to (19). Part (ii) of the lemma specifies that after a period of length $\ln (1 / \chi) / a$, the cost levels of the original problem are reached and the original entry problem is replicated.

Lemma 5 (iii) can be understood by considering the choice of units of time. Proportional changes in $a$ and $r$ are isomorphic to changes in time units, but the flow of profits is "thinner" when doubling the units of time. When the thinner stream of profits is compensated by a proportional increase in market size $L$, the total effect on the relative spacing of qualities vanishes. This statement also shows that for a fixed ratio of $r / a$ (e.g., if $r=a)$, the spacing property of $\gamma_{1}$ depends on the ratio $a / L$ only. Together with part (i) of the lemma, we can write $\gamma_{1}(a \phi /(\varphi L))$ in this case.

Finally, Lemma 5 (iv) shows that stretching or compressing the distribution of valuations $v$ does not affect equilibrium spacing. Stretching the distribution $G$ (with $\chi>1$ ) increases each consumer's willingness to pay for quality and thus simply advances the entry of firms. Together with (i) and (ii), this statement can be reformulated intuitively as an equivalence between the valuations $v$ and $\operatorname{costs} \phi$ and $\varphi$. A proportional increase in consumers' willingness to pay for quality and the costs of quality leaves the equilibrium qualities unchanged, and the entry date of each given quality occurs earlier by $\ln (1 / \chi) / a$ units of time.

We stress that Lemma 5 applies to the entry decision of any new entrant. It can thus be applied to the particular case of the ERSE, where $q_{n} / q_{n-1}=\bar{\gamma}$ for all $n \leq 0$ and $\gamma_{1}=\bar{\gamma}$ holds. In particular, we can write the ERSE parameter as a function of the ratio $\phi /(\varphi L)$ $\bar{\gamma}$. Our primary aim in this section is to conduct comparative statics with regard to this ratio of parameters that is key to the model.

With these preparatory steps, and with some parameter restrictions, we can sign the slope of the ERSE's location, i.e., the function $\bar{\gamma}(\phi)$.

Proposition 2 Assume that $\underline{v}=0$ and $(\theta, \varphi, L, r, a)$ is a combination of positive parameters. Then there is a $\phi_{o}>0$ such that $\bar{\gamma}(\phi)$ is weakly increasing on $\left[0, \phi_{o}\right]$ and strictly increasing for some $\phi \in\left[0, \phi_{o}\right]$.

Proof. See Appendix.

Proposition 2 establishes the comparative statics of the equilibrium degree of spacing 
with regard to the entry cost. ${ }^{24}$ In combination with Proposition 1 , it represents the main result of our analysis.

The proposition shows (at least within a restricted range of setup costs) that higher setup costs increase the relative spacing between quality levels. Intuitively, firms must be compensated for increases in setup costs by increased profits. The rise in profits is due to larger market shares, higher markups, and, ultimately, a wider spacing parameter $\bar{\gamma}$.

Together with Lemma 2, Proposition 2 also determines the impact of market size $(L)$ and marginal production costs $(\varphi)$ on the spacing $\bar{\gamma}$ of the ERSE. In particular, increases in $L$ and $\varphi$ have similar effects on $\bar{\gamma}$ as reductions in setup costs: all of these changes decrease the equilibrium spacing, $\bar{\gamma}$. Clearly, a larger market induces, ceteris paribus, higher profits and allows firms to generate more profit. Therefore, at given setup costs, larger markets experience more-frequent entry of firms at closer distances, i.e., the competitive pressure among firms rises.

Surprisingly, Proposition 2 suggests that higher productivity at the margin (a decrease in marginal production costs $\varphi$ ) increases relative spacing. This adverse effect of marginal productivity growth on competitive pressure may appear somewhat puzzling. To understand the forces operating to this effect, observe that the preference specification developed in this paper generates, just as with preferences featuring a constant elasticity of substitution, relative firm markups $p_{n} / c_{n}-1$ that are independent of costs (see prices (12)). In other words, at a given relative spacing, operating profits constitute a constant share of revenues. Hence, when quality levels are constant, an increase in marginal productivity (or a drop in marginal costs) tends to curb revenues and thereby depresses operating profits. $^{25}$ However, because firms must cover their setup costs, the productivity gains that curb profits per consumer must come about with increases in market share, i.e., with a wider equilibrium spacing. Simultaneously, this widening of relative spacing increases relative markups. Hence, competitive pressure decreases as marginal productivity grows. ${ }^{26}$

It would be premature to infer welfare consequences based on the parameter $\bar{\gamma}$ alone

\footnotetext{
${ }^{24}$ The condition on the interest rate $r$ establishes an upper bound for the instantaneous profits upon firm entry and is a technical requirement for the proof of Proposition 2.

${ }^{25}$ This aggregate relationship does not, of course, mean that each single firm can raise its profits by artificially decreasing its productivity.

${ }^{26}$ Note that this effect depends on the property of our model that demand does not react along an intensive margin. In particular, consumers do not react to price changes by consuming more or less but instead by switching to other firms.
} 
(and its impact on markups), conjecturing, e.g., that an equal increase in setup costs $\phi$ and operating costs $\varphi$ leaves the welfare levels of the economy unchanged. In fact, it does not. Such a change in technology actually postpones innovation by Lemma 5 (ii) so that more time elapses until a product of a given quality reaches the market. During this delay, individuals purchase lower-quality goods, with obviously negative consequences for consumer surplus.

\section{Conclusion}

In this paper, we analyze endogenous quality choice under costly entry into vertically differentiated markets. We do so assuming that production technology is such that the industry equilibrium can sustain a potentially unbounded number of active firms.

The firms' entry game consists of two stages: an optimal location choice and a subsequent price game. Proceeding through backward induction, we first derive pricing decisions, taking as given each firm's location in the quality space and assuming that firms engage in Bertrand competition. Our main contribution consists of the second step. Specifically, we model firm's costly entry and quality decisions in this industry. Exogenous productivity growth makes ever higher qualities affordable for consumers, so that firms sequentially enter the market at the top end of the quality spectrum. We specify the conditions under which the entry problem of each new entrant is replicated, which implies that each new quality level exceeds the current quality level by a fixed proportion. Although firms' sequential market entry introduces technical difficulties, this model overcomes the asymmetry of the location problem that unavoidably arises in quality space: the quality spectrum has top and bottom ends. Our technical contribution lies in handling these border conditions. 


\section{A Appendix - Proofs}

Proof that relative markups $\left(\lambda / \gamma^{\theta}\right)^{n} A+\alpha-1$ from (12) are positive. Use (13) to verify $\alpha>0 \Leftrightarrow 2(\gamma+1)>\gamma^{\theta}+\gamma^{1-\theta}$ and (17) to check $2(\gamma+1)=\lambda-\gamma / \lambda$. Together, this implies that the equivalence

$$
\alpha>0 \Leftrightarrow \lambda>\gamma^{\theta}
$$

holds.

Consider now the two cases $\alpha>0$ and $\alpha<0$. In first the case $\alpha>0, \alpha>1$ holds, as can be quickly checked by taking derivatives of $1 / \alpha$ with respect to $\gamma$ and evaluating $\alpha$ at $\gamma=1$. Hence, all relative markups are positive if $A \geq 0$. Alternatively, if $A<0$ the relative markups $\left(\lambda / \gamma^{\theta}\right)^{n} A+\alpha-1$ decrease in $n$ because of the equivalence (27). Hence, all relative markups are positive if and only if $A+\alpha-1>0$ holds.

Second, if $\alpha<0$, then $\left(\lambda / \gamma^{\theta}\right)^{n}$ decreases in $n$ and, again, all relative markups are positive if $A+\alpha-1>0$ holds.

It thus remains to show that $A+\alpha-1>0$ holds. But if this inequality does not hold, the top firm exits the market and, the effective top quality, $q_{0}$, would drop by $1 / \gamma$, thus increasing $A$ by (15) and (4). All the top firms exit the market up to the point when $A+\alpha-1>0$ holds.

Proof of Lemma 3. With (4), (13) and (15), rewrite (12) as

$$
p_{n}=\lambda^{n} \frac{\lambda}{2 \lambda-1}\left(-\frac{\gamma^{\theta}-\gamma^{-\theta}}{\gamma+1} \alpha c_{0}+\frac{\gamma-1}{\gamma} q_{0} \overline{\mathrm{v}}\right)+\alpha c_{n}
$$

where $c_{n}=\varphi q_{n}^{\theta}=\varphi \gamma^{n \theta} q_{0}^{\theta}$.

(i) Follows from expression (28) with $\gamma>1$ and $\lambda>1$.

(ii) Use (28) to compute

$$
\frac{d p_{n}}{d \varphi}=\left[-\lambda^{n} \frac{\lambda}{2 \lambda-1} \frac{\gamma^{\theta}-\gamma^{-\theta}}{\gamma+1} \alpha c_{0}+\alpha c_{n}\right] \frac{1}{\varphi}=\left[-\left(\frac{\lambda}{\gamma^{\theta}}\right)^{n} \frac{\lambda}{2 \lambda-1} \frac{\gamma^{\theta}-\gamma^{-\theta}}{\gamma+1}+1\right] \frac{c_{n} \alpha}{\varphi}
$$

If $\lambda>\gamma^{\theta}$, then the term in square brackets on the right hand side satisfies (recall $n \leq 0$ )

$$
-\left(\frac{\lambda}{\gamma^{\theta}}\right)^{n} \frac{\lambda}{2 \lambda-1} \frac{\gamma^{\theta}-\gamma^{-\theta}}{\gamma+1}+1 \geq-\frac{\lambda}{2 \lambda-1} \frac{\gamma^{\theta}-\gamma^{-\theta}}{\gamma+1}+1>-\frac{\lambda}{2 \lambda-1} \frac{\lambda-1 / \lambda}{\gamma+1}+1
$$

Since $\lambda$ solves (17), it satisfies $\lambda^{2}=2 \lambda(\gamma+1)-\gamma$ so that the expression on the right hand side is

$$
-\frac{1}{2 \lambda-1} \frac{\lambda^{2}-1}{\gamma+1}+1=-\frac{1}{2 \lambda-1} \frac{2 \lambda(\gamma+1)-\gamma-1}{\gamma+1}+1=-\frac{1}{2 \lambda-1} \frac{2 \lambda-1}{1}+1=0
$$


If, instead, $\lambda<\gamma^{\theta}$ then

$$
-\left(\frac{\lambda}{\gamma^{\theta}}\right)^{n} \frac{\lambda}{2 \lambda-1} \frac{\gamma^{\theta}-\gamma^{-\theta}}{\gamma+1}+1 \leq-\frac{\lambda}{2 \lambda-1} \frac{\gamma^{\theta}-\gamma^{-\theta}}{\gamma+1}+1<-\frac{\lambda}{2 \lambda-1} \frac{\lambda-1 / \lambda}{\gamma+1}+1
$$

The last expression is zero, as shown above.

By (27), $\alpha>0$ holds in the first case, while $\alpha<0$ in the second case. Hence, both cases together with (29) imply $d p_{n} / d \varphi \geq 0$.

Finally, observe with (12) and (15) that

$$
\frac{d p_{n}}{d q_{0}}=\frac{d p_{n}}{d \varphi} \frac{\varphi \theta}{q_{0}}+\lambda^{n} \frac{\lambda}{2 \lambda-1} \frac{\gamma-1}{\gamma} \overline{\mathrm{V}}
$$

This expression is positive because $d p_{n} / d \varphi \geq 0$.

(iii) Follows directly from (28), (4) and $\gamma>1$.

Proof of Lemma 4. First, denote the expression from (15) before (after) the entry of the additional firm with $A(\tilde{A})$. If $\tilde{A}+\alpha \leq 1$, the new entrant does not produce and there is no effect on prices. Thus, we assume wlog that $\tilde{A}+\alpha>1$ holds.

The main part of the proof consists of showing that $A-\widetilde{A} / \lambda>0$ holds. To that aim, set

$$
a=-\left[1-\alpha\left(2-\gamma^{-\theta}\right)\right] \quad \text { and } \quad b=\frac{\gamma-1}{\gamma} \frac{q_{0}}{c_{0}} \overline{\mathrm{v}}
$$

Collecting terms from (15) before and after entry of firm $n=1$, we need to show that $-a+b>-a \gamma^{\theta} / \lambda+b \gamma / \lambda$ or

$$
b>a \frac{\lambda-\gamma^{\theta}}{\lambda-\gamma}
$$

holds. In order to prove this inequality, a lower bound on $b$ is needed. To obtain it, observe that $\widetilde{A}+\alpha \gamma^{\theta} c_{0} \geq \gamma^{\theta} c_{0}$ (firm 1's markups cannot be negative), i.e. $\lambda /(2 \lambda-1)\left[-a \gamma^{\theta}+b \gamma\right] \geq$ $\gamma^{\theta}(1-\alpha)$ holds, or

$$
b \geq \gamma^{\theta-1}\left[\frac{2 \lambda-1}{\lambda}(1-\alpha)+a\right]
$$

For claim $A-\widetilde{A} / \lambda$ to hold, it is thus sufficient to show

$$
\gamma^{\theta-1} \frac{2 \lambda-1}{\lambda}(1-\alpha)+a \gamma^{\theta-1}>a \frac{\lambda-\gamma^{\theta}}{\lambda-\gamma}
$$

or

$$
\gamma^{\theta-1} \frac{2 \lambda-1}{\lambda}(1-\alpha)>a \lambda \frac{1-\gamma^{\theta-1}}{\lambda-\gamma}
$$


Observing that $\lambda$ solves $\lambda^{2}-2(\gamma+1) \lambda+\gamma=0$, we get $\lambda^{2}-2 \lambda=(2 \lambda-1) \gamma$ so that the above inequality can be rewritten as

$$
\gamma^{\theta-1}(\lambda-2)(1-\alpha)>a \lambda \gamma \frac{1-\gamma^{\theta-1}}{\lambda-\gamma}
$$

With $a=-\left[1-\alpha\left(2-\gamma^{-\theta}\right)\right]$ as defined above, it is equivalent

$$
\gamma^{\theta-1}(\lambda-2)(\lambda-\gamma)(\alpha-1)<\left[1-\alpha\left(2-\gamma^{-\theta}\right)\right] \lambda \gamma\left(1-\gamma^{\theta-1}\right)
$$

Notice that $(\lambda-2)(\lambda-\gamma)=\left[\lambda^{2}-2(1+\gamma) \lambda+\gamma\right]+\gamma+\gamma \lambda=\gamma(\lambda+1)$ so that we need to show that

$$
\gamma^{\theta-1}(\lambda+1)(\alpha-1)<\left[\alpha\left(2-\gamma^{-\theta}\right)-1\right] \lambda\left(\gamma^{\theta-1}-1\right)
$$

holds. After rearranging and using $\alpha$ from (13), this inequality becomes is equivalent to

$$
0<\left(\gamma^{\theta-1}+\lambda\right)\left(2(\gamma+1)-\gamma^{\theta}-\gamma^{1-\theta}\right)-(\gamma+1)\left\{\gamma^{\theta-1}(\lambda+1)+\left(2-\gamma^{-\theta}\right) \lambda\left(1-\gamma^{\theta-1}\right)\right\}
$$

The expression of the right hand side is

$$
\begin{aligned}
\ldots= & \lambda\left[2(\gamma+1)-\gamma^{\theta}-\gamma^{1-\theta}-(\gamma+1)\left\{\gamma^{\theta-1}+\left(2-\gamma^{-\theta}\right)\left(1-\gamma^{\theta-1}\right)\right\}\right]+\ldots \\
& \ldots+\gamma^{\theta-1}\left((\gamma+1)-\gamma^{\theta}-\gamma^{1-\theta}\right) \\
= & \lambda\left[\gamma\left(\gamma^{\theta}+\gamma^{1-\theta}\right)-(\gamma+1) / \gamma\right]-\gamma^{\theta-1}\left(\gamma^{\theta}+\gamma^{1-\theta}-(\gamma+1)\right) \\
= & \left(\lambda \gamma-\gamma^{\theta-1}\right)\left(\gamma^{\theta}+\gamma^{1-\theta}\right)-(\gamma+1)\left[\lambda-\gamma^{\theta}\right] / \gamma
\end{aligned}
$$

Since $\lambda>\gamma^{\theta-1}$ (from (27)) and $\gamma^{\theta}+\gamma^{1-\theta}>\gamma+1$ (as quickly checked) the last term exceeds

$$
\left[\left(\lambda \gamma-\gamma^{\theta-1}\right)-\left[\lambda-\gamma^{\theta}\right] / \gamma\right](\gamma+1)=[\lambda \gamma-\lambda / \gamma](\gamma+1)
$$

and is thus positive. This proves that $A-\widetilde{A} / \lambda>0$.

(i) Denoting the price of firm $k$ before and after entry of the additional firm with $p_{k}$ and $\tilde{p}_{k}$, respectively, we have

$$
p_{k}-\tilde{p}_{k}=A \lambda^{k}+\alpha c_{k}-\left(\widetilde{A} \lambda^{k-1}+\alpha c_{k}\right)=\lambda^{k}(A-\widetilde{A} / \lambda)
$$

The term in brackets is positive as shown above, proving the claim.

(ii) We compute

$$
\left(\tilde{p}_{k+1}-\tilde{p}_{k}\right)-\left(p_{k+1}-p_{k}\right)=\left(\lambda^{k+1-1}-\lambda^{k-1}\right) \widetilde{A}-\left(\lambda^{k+1}-\lambda^{k}\right) A \leq 0 .
$$


where the last inequality follows as above.

(iii) This statement holds by (ii) and (5).

Proof of Proposition 1. (i) Without loss of generality, we consider the timing and location choice of the entrant $n=1$, given $q_{0}$ and $\left\{\gamma_{n}\right\}_{n \leq 0}$, where $\gamma_{n}=q_{n} / q_{n-1}$.

Consider first the re-normalization of time $t^{\prime}=t+(\theta-1) a^{-1} \ln \left(1 / q_{0}\right)$, which must leave real choices unchanged. Check then with (19) that under this time re-normalization, marginal and setup costs satisfy $\varphi_{t^{\prime}}=\varphi_{t} q_{0}^{\theta-1}$ and $\phi_{t^{\prime}}=\phi_{t} q_{0}^{\theta-1}$. Hence, applying Lemma 1 to the specific transformation $\left(q_{0}, t\right) \rightarrow\left(1, t^{\prime}\right)$, the operating profit of all firms $m$ satisfy $\pi_{m}^{\prime}=\pi_{m} / q_{0}$. Moreover, entry costs under this transformation are $\phi_{t^{\prime}}=\phi_{t} q_{0}^{\theta} / q_{0}$, i.e. scaled by the factor $1 / q_{0}$, too. This implies that under the above transformation, the problem of quality choice by potential entrants is scaled by the factor $1 / q_{0}$ and the optimal $\gamma_{1}$ is independent of $q_{0}$. Without loss of generality, we thus set $q_{0}=1$.

Next, we show that the optimal entry date $t_{1}$ is finite. Observe that firm 1 's profits are bounded above by profits that accrue in the absence of any competitors. In this hypothetical case the monopolist sells to the market segment $\left[p^{m o n} / q, \bar{v}\right]$, charging the optimal price $p^{m o n}=\max \left\{\left(\overline{\mathrm{v}} \gamma_{1}+c_{1}\right) / 2, \underline{\mathrm{v}} \gamma_{1}\right\}$ and receiving profits according to (8)

$$
\pi^{m o n}(\tau)=\left(\max \left\{0, p^{m o n}-c_{1}\right\}\right)^{2} L /\left(\gamma_{1}(\overline{\mathrm{v}}-\underline{\mathrm{v}})\right)
$$

Recalling (19), we define $\underline{\mathrm{t}}$ as the finite $t$ where $p^{\text {mon }}=c_{1}$ holds and notice that $\pi^{\text {mon }}(\tau)=0$ if $\tau<\underline{\mathrm{t}}$. The net present value of these profits for entry at time $t_{1}$ is then

$$
\frac{L}{\gamma_{1}(\overline{\mathrm{v}}-\underline{\mathrm{v}})} \int_{\max \left(t_{1}, \underline{\mathrm{t}}\right)}^{\infty} e^{-r\left(\tau-t_{1}\right)}\left(\max \left\{\left(\bar{v} \gamma_{1}+\varphi e^{-a \tau} \gamma_{1}^{\theta-1}\right) / 2, \underline{\mathrm{v}} \gamma_{1}\right\}-\varphi e^{-a \tau} \gamma_{1}^{\theta-1}\right)^{2} d \tau-\phi e^{-a t_{1}} \gamma_{1}^{\theta}
$$

The integral is zero at $t_{1} \rightarrow-\infty$ while entry costs $\phi e^{-a t_{1}}$ grow unbounded. Thus, the entire expression is negative, contradicting optimal entry. In sum, we have $-\infty<t_{1}<\infty$, and $t_{1}$ is unique by virtue of the infimum.

Next, we show the optimal choice $\gamma$ satisfies $\gamma \in(1, \infty)$. Entry at $\gamma_{1}=1$ is not optimal since Bertrand competition would imply $\pi^{1}=0$ so that firms do not recover positive entry costs. To show that entry at $\gamma_{1} \rightarrow \infty$ is not optimal, use again the fact that operating profits are bounded above by those in absence of all competitors. Thus, using the expression for $\pi^{m o n}(\tau)$ above and setting $\varphi=0$, we compute an upper bound for firm 1's net present value of profits:

$$
(\max \{\overline{\mathrm{v}} / 2, \underline{\mathrm{v}}\})^{2} \gamma_{1} L /(\overline{\mathrm{v}}-\underline{\mathrm{v}}) \int_{t_{1}}^{\infty} e^{-r \tau} d \tau-\phi e^{-a t_{1}} \gamma_{1}^{\theta}
$$


This expression is negative for $\gamma_{1} \rightarrow \infty$. Thus, net profits are maximal for a $\gamma_{1} \in(1, \infty)$. For given $t_{1}$, the global maximum of net present value of profits global maximum in $\gamma_{1}$ is then unique except for knife-edge cases, as in generic maximization problems with finite support.

(ii) The proof is done by induction through $n$. Assume that $\left\{q_{n}\right\}_{n \leq 0}$ satisfy (10) with prevailing $\gamma$ and consider the entry decision of firm $m=1$. Observe that entry with $\gamma_{1}=1$ is not optimal because, as shown in part (i) above, Bertrand competition would imply $\Pi_{t_{1}}\left(\gamma_{1}\right)=0$ (regardless of $t_{1}$ ) and thus violate the free entry condition. Hence, $\gamma=1$ implies $\gamma_{1}>\gamma$.

Next, we show that $\gamma_{1}<\gamma$ holds for $\gamma$ large enough. Assume this is not the case: $\gamma_{1} \geq \gamma$ for all $\gamma \geq 1$, which implies $\gamma_{1} \rightarrow \infty$ as $\gamma \rightarrow \infty$. But $\gamma_{1} \rightarrow \infty$ was shown in part (i) to violate free entry. Consequently, $\gamma_{1}<\gamma$ holds for $\gamma$ large enough.

Writing now the first entrant's relative location $\gamma_{1}$ as a function of the pre-existing relative location parameter $\gamma$, i.e., writing $\gamma_{1}(\gamma)$ we thus have $\gamma_{1}(\gamma)>\gamma$ for $\gamma=1$ and $\gamma_{1}(\gamma)<\gamma$ for $\gamma$ large enough. Continuity then implies that there is a $\gamma>1$ so that $\gamma_{1}=\gamma$. We denote this value by $\bar{\gamma}$. At $\gamma=\bar{\gamma}$, the firm $n=1$ locates in the quality space, extending equal relative spacing (10) to all $n \leq 1$.

Under $\gamma=\gamma_{1}=\bar{\gamma}$, we call the spacing problem of the remaining additional firms $(n=$ $2,3, \ldots)$ the residual spacing problem. With the notation

$$
\gamma_{n}^{\prime}=\gamma_{n+1} \quad(n \geq 1) \quad q_{0}^{\prime}=\bar{\gamma} q_{0}=q_{1} \quad \text { and } \quad \tau^{\prime}=\tau+a^{-1}(\theta-1) \ln \bar{\gamma}
$$

the residual spacing problem solves the corresponding system (22) - (24) above, where the relevant variables $q^{\prime}, \tau^{\prime}, t_{m}^{\prime}$ and $\gamma^{\prime}$ now bear a prime. An induction argument completes the proof that $\gamma_{n} \equiv \bar{\gamma}$ for all $n \geq 1$.

Finally, (19) and the transformation (30) show that two consecutive entries occur at dates satisfying $\phi_{t_{n}}=\bar{\gamma}^{1-\theta} \phi_{t_{n}}$. With (19), this is $e^{-a\left(t_{n+1}-t_{n}\right)}=\bar{\gamma}^{1-\theta}$ and proves (25).

Proof of Proposition 2. We begin the proof by defining $\Psi(\hat{\gamma}, t, \gamma, \phi)$ as the net present value of profits of the first entrant (firm $m=1$ ). This value is expressed as a function of existing spacing $\gamma_{n}=\gamma$ for all $n \leq 0$, parameter $\phi$ of setup costs (19), entry date $t$ and location choice $\hat{\gamma}$. Normalizing $q_{0}=1$ we have

$$
\Psi(\hat{\gamma}, t, \gamma, \phi)=\Pi(\hat{\gamma}, t, \gamma)-\phi e^{-a t}(\hat{\gamma})^{\theta}
$$


where $\Pi(\hat{\gamma}, t, \gamma)$ is as defined in (21). We will denote the partial derivatives of $\Psi$ w.r.t the four arguments as $\Psi_{\hat{\gamma}}, \Psi_{t}, \Psi_{\gamma}$ and $\Psi_{\phi}$, respectively. Free entry implies that firm 1's equilibrium entry date and location, which we denote by $t^{*}(\gamma)$ and $\gamma^{*}(\gamma)$, satisfy

$$
\Psi\left(\gamma^{*}(\gamma), t^{*}(\gamma), \gamma, \phi\right)=0
$$

for all $\gamma$ and $\phi$. Optimality of the firm's location choice implies

$$
\Psi_{\hat{\gamma}}\left(\gamma^{*}(\gamma), t^{*}(\gamma), \gamma, \phi\right)=0
$$

Taking derivatives of (32) w.r.t. $\gamma$ and using (33) yields

$$
\Psi_{t} \frac{d t^{*}}{d \gamma}+\Psi_{\gamma}=0
$$

We now denote the relative spacing variable of the ERSE with $\bar{\gamma}(\phi)$. Along the path of the ERSEs, identity (32) holds, i.e., $\Psi\left(\bar{\gamma}(\phi), t^{*}(\bar{\gamma}(\phi), \phi), \bar{\gamma}(\phi), \phi\right)=0$. Taking derivatives w.r.t. $\phi$ and using (33) and (34) yields

$$
0=\left[\Psi_{t} \frac{d t^{*}}{d \gamma}+\Psi_{\gamma}\right] \frac{d \bar{\gamma}}{d \phi}+\Psi_{t} \frac{\partial t^{*}}{\partial \phi}+\Psi_{\phi}=\Psi_{t} \frac{\partial t^{*}}{\partial \phi}+\Psi_{\phi}
$$

where $\partial t^{*} / \partial \phi$ is the partial derivative of the entry date w.r.t. entry costs, when existing spacing is held constant $\left(\gamma_{n}=\bar{\gamma}\right)$ and $d t^{*} / d \gamma$ is the derivative of the entry date w.r.t $\bar{\gamma}$ the parameter of existing spacing, when entry costs are held constant. With $\Psi_{\phi}=-e^{-a t^{*}} \bar{\gamma}^{\theta}$, the last equation implies

$$
\frac{\partial t^{*}}{\partial \phi}=\frac{e^{-a t^{*}} \bar{\gamma}^{\theta}}{\Psi_{t}}
$$

Now, taking derivatives of (33) w.r.t. $\gamma$ leads to

$$
0=\Psi_{\hat{\gamma} \hat{\gamma}} \frac{d \gamma^{*}}{d \gamma}+\Psi_{\hat{\gamma} t} \frac{d t^{*}}{d \gamma}+\Psi_{\hat{\gamma} \gamma}
$$

Along the path of all ERSE, (33) applies, i.e., $\Psi_{\hat{\gamma}}\left(\bar{\gamma}(\phi), t^{*}(\bar{\gamma}(\phi), \phi), \bar{\gamma}(\phi), \phi\right)=0$. Taking derivatives w.r.t. $\phi$ yields

$$
0=\left[\Psi_{\hat{\gamma} \hat{\gamma}}+d \Psi_{\hat{\gamma} t} \frac{d t^{*}}{d \gamma}+\Psi_{\hat{\gamma} \gamma}\right] \frac{d \bar{\gamma}}{d \phi}+\Psi_{\hat{\gamma} t} \frac{\partial t^{*}}{\partial \phi}+\Psi_{\hat{\gamma} \phi}
$$

and with (36)

$$
\Psi_{\hat{\gamma} \hat{\gamma}}\left[1-\frac{d \gamma^{*}}{d \gamma}\right] \frac{d \bar{\gamma}}{d \phi}=-\Psi_{\hat{\gamma} t} \frac{\partial t^{*}}{\partial \phi}-\Psi_{\hat{\gamma} \phi}
$$


Equations (3), (19), (21) and (31) imply $\Psi_{t}=r \phi e^{-a t}(\gamma)^{\theta}-\pi(t)+a \phi e^{-a t}(\gamma)^{\theta}$ and $\Psi_{\hat{\gamma} t}=$ $(a+r) \phi e^{-a t} \theta(\gamma)^{\theta-1}-\pi_{\hat{\gamma}}(t)$. Further, observe that $\Psi_{\hat{\gamma} \phi}=-\theta e^{-a t}(\gamma)^{\theta-1}$ holds. Combining these expressions with (35) yields

$$
\Psi_{\hat{\gamma} \hat{\gamma}}\left[1-\frac{d \gamma^{*}}{d \gamma}\right] \frac{d \bar{\gamma}}{d \phi}=\left\{\pi_{\hat{\gamma}}\left(t^{*}\right)-\pi\left(t^{*}\right) \theta / \bar{\gamma}\right\} \frac{\bar{\gamma}^{\theta} e^{-a t^{*}}}{\Psi_{t}}
$$

The second order condition of the firm's optimization problem requires $\Psi_{\hat{\gamma} \hat{\gamma}}<0$, while (26) implies that the term in the square brackets is positive. Moreover, by definition of $t^{*}$, $\Psi_{t}>0$ holds at equilibrium entry. Consequently, $\bar{\gamma}(\phi)$ is increasing in $\phi$ if and only if the expression in the slanted brackets on the right of (37) is negative. This condition can be reformulated as

$$
\frac{d}{d q_{1}} \ln \left(\pi\left(t^{*}\right)\right)<\theta / \bar{\gamma}
$$

We now use profits $\pi_{1}=L\left(p_{1}-c_{1}\right)^{2} /\left(\overline{\mathrm{v}}\left(q_{1}-q_{0}\right)\right)$ (compare (8) and (9), shifting indices), to rewrite condition (38) as

$$
2 \frac{\dot{p}_{1}-\dot{c}_{1}}{p_{1}-c_{1}}<\frac{\theta}{q_{1}}+\frac{1}{q_{1}-q_{0}}
$$

where $\dot{x} \equiv d x / d q_{1}$. To derive an expression of $\dot{p}_{1}$, write the system (11) as

$$
\left(\begin{array}{ccccc}
2 & -1 & 0 & 0 & \ldots \\
-\left(q_{0}-q_{-1}\right) & 2\left(q_{1}-q_{-1}\right) & -\left(q_{1}-q_{0}\right) & 0 & \\
0 & -\left(q_{-1}-q_{-2}\right) & 2\left(q_{0}-q_{-2}\right) & \ldots & \\
\cdots & 0 & \cdots & \cdots & \ldots
\end{array}\right) p=\left(\begin{array}{c}
c_{1}+\left(q_{1}-q_{0}\right) \overline{\mathbf{v}} \\
\left(q_{1}-q_{-1}\right) c_{0} \\
\left(q_{0}-q_{-2}\right) c_{-1} \\
\cdots
\end{array}\right)
$$

where $p \equiv\left(p_{1}, p_{0}, \ldots\right)^{t}$. Taking derivatives w.r.t. $q_{1}$ yields

$$
\left(\begin{array}{cccc}
2 & -1 & 0 & 0 \\
-\left(q_{0}-q_{-1}\right) & 2\left(q_{1}-q_{-1}\right) & -\left(q_{1}-q_{0}\right) & 0 \\
0 & -\left(q_{-1}-q_{-2}\right) & 2\left(q_{0}-q_{-2}\right) & \ldots \\
\ldots & 0 & \ldots & \ldots
\end{array}\right) \dot{p}+\left(\begin{array}{ccccc}
0 & 0 & 0 & 0 & \ldots \\
0 & 2 & -1 & 0 & \\
0 & 0 & 0 & 0 & \\
\ldots & & & & \ldots
\end{array}\right) p=\left(\begin{array}{c}
\dot{c}_{1}+\overline{\mathrm{v}} \\
c_{0} \\
0 \\
\ldots
\end{array}\right)
$$

Evaluating at the ERSE and setting $q_{0}=1$ leads to

$$
\left(\begin{array}{ccccc}
2 & -1 & 0 & 0 & \cdots \\
-1 & 2(\gamma+1) & -\gamma & 0 & \\
0 & -1 & 2(\gamma+1) & -\gamma & \\
\cdots & 0 & \cdots & \cdots & \cdots
\end{array}\right) \dot{p}=\left(\begin{array}{c}
\dot{c}_{1}+\overline{\mathrm{v}} \\
\gamma \frac{-2 p_{0}+p_{-1}+c_{0}}{\gamma-1} \\
0 \\
\ldots
\end{array}\right)
$$

Replicating the proof of Lemma 2, we obtain that $\dot{p}_{n}$ satisfies $\dot{p}_{n}=\lambda^{n} \dot{p}_{0}$ for $n \leq 0$ with $\lambda=\gamma+1+\sqrt{\gamma^{2}+\gamma+1}$. Thus, the second row of (41) is

$$
-\dot{p}_{1}+2(\gamma+1) \dot{p}_{0}-\gamma \lambda^{-1} \dot{p}_{0}=-\dot{p}_{1}+\lambda \dot{p}_{0}=\gamma \frac{-2 p_{0}+p_{-1}+c_{0}}{\gamma-1}
$$


where the first identity follows because $\lambda$ solves (17). With the first row of (41) $\left(2 \dot{p}_{1}-\dot{p}_{0}=\right.$ $\left.\dot{c}_{1}+\overline{\mathrm{v}}\right)$, this yields

$$
(2 \lambda-1) \dot{p}_{1}=\lambda\left[\dot{c}_{1}+\overline{\mathrm{v}}\right]+\gamma \frac{-2 p_{0}+p_{-1}+c_{0}}{q_{1}-q_{0}}
$$

and after rearranging and using the second row of (40)

$$
(2 \lambda-1)\left(\dot{p}_{1}-\dot{c}_{1}\right)=\lambda\left(\overline{\mathrm{v}}-\dot{c}_{1}\right)+\dot{c}_{1}+\frac{2 p_{0}-p_{1}-c_{0}}{\gamma-1}
$$

Now distinguish the two cases $\lim _{\phi \rightarrow 1} \bar{\gamma}(\phi)=1$ and $\lim _{\phi \rightarrow 1} \bar{\gamma}(\phi)>1$. In the first case, we observe with $\bar{\gamma}(0)=1$ and $\bar{\gamma}(\phi)>1$ for all $\phi>0$ that there is a $\phi_{0}$ so that $\bar{\gamma}(\phi)$ is increasing for $\phi<\phi_{o}$. In the second of the cases, we observe that $\bar{\gamma}(\phi)>0$ implies $\pi_{1}(t)>0$ for $t$ large enough so that, in order for the free entry condition (32) to be satisfied, $t_{1}(\phi) \rightarrow-\infty$ at $\phi \rightarrow 0$ must hold. This, in turn, implies that $\bar{\gamma}(\phi) \overline{\mathrm{v}}<c_{1}(\phi)=e^{-a t_{1}(\phi)}[\bar{\gamma}(\phi)]^{\theta}$ for $\phi$ small enough so that demand is zero upon entry for these $\phi$. We define the maximum of the set of such $\phi$ as $\phi_{o}$ and observe that, by construction, $\bar{\gamma}\left(\phi_{o}\right)$ and $t^{*}\left(\phi_{o}\right)$ satisfy conditions (22) and (23) of optimal entry for firm 1 , given $\gamma_{n}=\bar{\gamma}(\phi)$ for all $n \leq 0$. When defining the entry date $t^{*}(\phi)=t^{*}\left(\phi_{o}\right)+a^{-1} \ln \left(\phi / \phi_{o}\right)$ and the location $\bar{\gamma}(\phi)=\bar{\gamma}\left(\phi_{o}\right)$ for all $\phi \leq \phi_{o}$, these choices $\bar{\gamma}\left(\phi_{o}\right)$ and $t^{*}\left(\phi_{o}\right)$ satisfy conditions (22) and (23) for firm 1 as well, given that $\gamma_{n}=\bar{\gamma}(\phi)$ for all $n \leq 0$. Hence, $\bar{\gamma}(\phi)=\bar{\gamma}\left(\phi_{o}\right) \geq 1$ for all $\phi \leq \phi_{o}$ holds. Now observe that at $\phi=\phi_{o}, p_{1}=c_{1}$ holds so that condition (39) becomes

$$
\dot{p}_{1}-\dot{c}_{1}<0
$$

But $p_{1}=c_{1}$ also implies $\overline{\mathrm{v}}=v_{1}$ by $(5)$ and $(7)$, or $\overline{\mathrm{v}}=\left(c_{1}-p_{0}\right) /(\gamma-1)$, so that we have with (42)

$$
\begin{aligned}
(2 \lambda-1)\left(\dot{p}_{1}-\dot{c}_{1}\right) & =(\lambda-1)\left(\frac{c_{1}-p_{0}}{\gamma-1}-\dot{c}_{1}\right)+\frac{p_{0}-c_{0}}{\gamma-1} \\
& =(\lambda-1)\left(\frac{c_{1}-c_{0}}{\gamma-1}-\dot{c}_{1}\right)+(\lambda-2)\left(\frac{c_{0}-p_{0}}{\gamma-1}\right)
\end{aligned}
$$

The last expression is negative, because $\lambda>2, p_{0}>c_{0}$ and, by concavity of $c_{1}$ in $\gamma$, $\dot{c}_{1}>\left(c_{1}-c_{0}\right) /(\gamma-1)$. This implies that condition (38) is satisfied and hence that the right hand side derivative of $\bar{\gamma}$ at $\phi_{o}\left(\lim _{\varepsilon \rightarrow 0}(\bar{\gamma}(|\varepsilon|)-\bar{\gamma}(0)) /|\varepsilon|\right)$ is strictly positive. By continuity there is a $\delta>0$ so that $\bar{\gamma}(\phi)$ is strictly increasing for $\phi \in\left[\phi_{0}, \phi_{0}+\delta\right]$. This completes the proof. 


\section{References}

[1] Aizcorbe, Ana and Samuel Kortum (2005). "Moore's Law and the Semiconductor Industry: A Vintage Model," Scandinavian Journal of Economics, Blackwell Publishing, vol. 107(4), pages 603-630, December.

[2] Aghion, Philippe and Peter Howitt (1992). "A Model of Growth Through Creative Destruction", Econometrica, Vol. 60, No. 2 (Mar., 1992), pp. 323-351.

[3] Anderson, Simon P. and Jacob K. Goeree (1997). "Location, Location, Location," Journal of Economic Theory, Vol. 77, pp. 102-127.

[4] Anderson, Simon P., André De Palma, Jacques-François Thisse (1989). Demand for differentiated products, discrete choice models, and the characteristics approach. Review of Economic Studies, Vol. 56.1, pp. 21-35.

[5] Anderson, Simon P., André De Palma, Jacques-François Thisse (1992) Discrete choice theory of product differentiation, MIT Press, 1992, Cambridge, MA.

[6] Auer, Raphael A, Thomas Chaney, and Philip Sauré. (2014). "Quality Pricing-toMarket." CEPR Discussion Papers No. 10053, July 2014.

[7] Baldwin, R. and J. Harrigan (2007). "Zeros, quality and space: Trade theory and trade evidence." American Economic Journal: Microeconomics, Vol 3 (May 2011):6088.

[8] Bems, Rudolfs \& Di Giovanni, Julian, 2014. "Income-Induced Expenditure Switching." CEPR Discussion Papers 9887, C.E.P.R. Discussion Papers.

[9] Bils, M and Klenow, PJ (2001). "Quantifying quality growth" The American Economic Review, 91 (4): 1006-1030, September 2001.

[10] Biscaia, Ricardo and Isabel Mota (2012). "Models of spatial competition: A critical review" Papers in Regional Science (forthcoming).

[11] Bresnahan, T. F. (1980). "Three Essays on the American Automobile Oligopoly." Unpublished PhD Dissertation, Princeton University 
[12] Bresnahan, Timothy F. (1987) "Competition and collusion in the American automobile industry: The 1955 price war." Journal of Industrial Economics pp. 457-482.

[13] Broda, Christian, and John Romalis. (2009). "The Welfare Implications of Rising Price Dispersion." Manuscript, Univ. Chicago.

[14] CAplin, Andrew, and Barry Nalebuff (1991). Aggregation and imperfect competition: On the existence of equilibrium. Econometrica, pp. 25-59.

[15] Capozza, Dennis R. and Robert Van Order (1980), "Unique Equilibria, Pure Profits, and Efficiency in Location Models." The American Economic Review, Vol. 70, No. 5, pp. 1046-1053.

[16] Champsaur P and Rochet JC (1989), "Multiproduct Duopolists", Econometrica, 57(3), 533-557, May 1989.

[17] Chor, Davin and Edwin L.C. LAI (2013) "Cumulative Innovation, Growth and Welfare Improving Patent Policy", Mimeo, Singapore Management University, February 2013.

[18] Cooper, Russell and Andrew John "Coordinating Coordination Failures in Keynesian Models", The Quarterly Journal of Economics, Vol. 103, No. 3 (Aug., 1988), pp. 441-463.

[19] D'Aspremont, C., J. J. Gabszewicz, and J.-F Thisse (1979): "On Hotelling's 'Stability in Competition'," Econometrica Vol. 47, pp. 1145-1150.

[20] Dhingra, Swati, and John Morrow (2014). "Monopolistic Competition and Optimum Product Diversity Under Firm Heterogeneity" LSE mimeo

[21] Debreu, G. (1952), "A Social Equilibrium Existence Theorem", Proceedings of the National Academy of Sciences, Vol. 38, pp. 886-893.

[22] Economides, N (1989). "Symmetric Equilibrium Existence and Optimality in Differentiated Product Markets" Journal of Economic Theory, 47 (1): 178-194 
[23] Evgeny, Z., S. Kokovin, M. Parenti, and J.-F. Thisse 2012. "Monopolistic Competition: Beyond the Constant Elasticity of Substitution," Econometrica, 80(6), pp. 2765-2784.

[24] Fajgelbaum, Pablo, Gene M. Grossman, and Elhanan Helpman, 2011. "Income Distribution, Product Quality, and International Trade." Journal of Political Economy, University of Chicago Press, vol. 119(4), pages 721 - 765

[25] Foellmi, R. and Zweimueller 2006 "Income distribution and demand-induced innovations" Review of Economic Studies, 73(4), 941-960

[26] Gabszewicz, Jaskold and Thisse, J.-F. (1979), "Price competition, quality and income disparities" Journal of Economic Theory, 20(3), pp.340-359, June 1980.

[27] Glicksberg, I. L. (1952), "A Further Generalization of the Kakutani Fixed Point Theorem with Application to Nash Equilibrium Points", Proceedings of the American Mathematical Society Vol. 38, pp. 170-174.

[28] _-_- (1980), "Entry (And Exit) in a Differentiated Industry" Journal of Economic Theory, 22 (April, 1980), 327-338.

[29] Golub, G. H., and Van Loan, C. F. (2012). "Matrix computations" (Vol. 3). JHU Press.

[30] Grossman, G. and E. Helpman (1991). "Quality ladders and product cycles." The Quarterly Journal of Economics 106, 557-586.

[31] Hallak, Juan Carlos, (2006). "Product quality and the direction of trade," Journal of International Economics, Elsevier, vol. 68(1), pages 238-265, January.

[32] Hallak, Juan Carlos and P. SchotT (2011). "Estimating cross-country differences in product quality". The Quarterly Journal of Economics, vol. 126(1), pages 417-474.

[33] Hotelling, Harold (1929), "Stability in Competition", Economic Journal, 39 (153): pages $41-57$.

[34] Johnson, R. (2012). "Trade and prices with heterogeneous firms." Journal of International Economics, vol 86 (1). 
[35] Khandelwal, Amit (2010) "The Long and Short (of) Quality Ladders," The Review of Economic Studies, 77(4), 1450-1476, 2010.

[36] Klette, TJ and Kortum, S "Innovating firms and aggregate innovation", The Journal of Political Economy, 112 (5): 986-1018 OCT 2004

[37] Kugler, Maurice and Eric Verhoogen, (2010). "Prices, Plant Size, and Product Quality." With Maurice Kugler. Mimeo Columbia University, July 2010

[38] Lahmandi-Ayed, R. 2000, "Natural oligopolies: A vertical differentiation model" International Economic Review, 41 (4): 971-987, November 2000.

[39] -. (2004). "Finiteness property in vertically differentiated markets: a note on locally increasing and decreasing returns" Economic Theory, 23 (2): 371-382, January 2004.

[40] Lancaster, Kelvin J. (1966). "A New Approach to Consumer Theory." The Journal of Political Economy, 74, 132-157.

[41] Moтta, Massimo. (1993): "Endogenous quality choice: price vs. quantity competition." Journal of Industrial Economics, 41 (2), 113-131.

[42] Mussa, Michael, and Sherwin Rosen. (1978). "Monopoly and Product Quality." The Journal of Economic Theory, 18 (2): 301-317.

[43] Neven, Damien J. 1986: "On Hotelling's Competition with Non-Uniform Consumer Distributions" Economics Letters Vol. 21, pp. 121-126.

[44] Osborne, M. J. and C. Pitchik 1987: “Equilibrium in Hotelling's Model of Spatial Competition," Econometrica 55 (4), pp. 911-922.

[45] Reny, Philip J. 1999: "On the Existence of Pure and Mixed Strategy Nash Equilibria in Discontinuous Games.” Econometrica, Vol. 67(5), pp. 1029-1056.

[46] Rosen, J. B. (1965). "Existence and Uniqueness of Equilibrium Points for Concave N-Person Games", Econometrica, Vol. 33, No. 3 (Jul., 1965), pp. 520-534.

[47] Salop, Steven C. (1979). "Monopolistic Competition with Outside Goods", The Bell Journal of Economics, Vol. 10, No. 1 (Spring, 1979), 141-156. 
[48] Schотт, Peter K. (2004). "Across-Product versus Within-Product Specialization in International Trade", Quarterly Journal of Economics, 119(2):647-678.

[49] Shaked, Avner, and John Sutton. (1982). "Relaxing Price Competition Through Product Differentiation." Review of Economic Studies, 49: 3-13.

[50] Shaked, Avner, and John Sutton. (1983). "Natural Oligopolies." Econometrica, 51 (5): 1469-1483.

[51] Shaked, Avner, and John Sutton. (1984). "Natural Oligopolies and International Trade." in (H. Kierzkowski ed.), Monopolistic Competition and International Trade, pp. 34-50, Oxford: Oxford University Press.

[52] Sutton, John (1986) "Vertical Product Differentiation - Some Basic Themes" The American Economic Review, 76 (2): 393-398 MAY 1986".

[53]_-_ (1996) "Technology and Market Structure." European Economic Review, Vol. 40 (1996), pp. 511-530.

[54] _- (1998) "Technology and Market Structure: Theory and History." Cambridge, Mass.: MIT Press, 1998.

[55] _- (2007). "Market Structure: Theory and Evidence." in (M. Armstrong and R. Porter, eds.), Handbook of Industrial Organization, vol. III, pp. 2301-68, Amsterdam: Elsevier.

[56] Tirole, Jean (1992). "The Theory of Industrial Organization" MIT Press, 1992

[57] Verhoogen, Eric (2008). "Trade, quality upgrading, and wage inequality in the Mexican manufacturing sector". The Quarterly Journal of Economics 123, 489-530.

[58] Vives, Xavier. 1988. "Sequential entry, industry structure and welfare," European Economic Review, Elsevier, vol. 32(8), pages 1671-1687, October.

[59] _-_ Oligopoly pricing: old ideas and new tools. MIT press, 2001.

[60] VogeL, John (2008). "Spatial Competition with Heterogeneous Firms" The Journal of Political Economy, Volume 116, Number 3, June 2008. 
[61] World Intellectual Property Organization, (2004). "WiPO Intellectual Property Handbook: Policy, Law and Use." WIPO Publication No. 489 (E).

[62] Zweimueller, Josef, and Johann K. Brunner (2005). "Innovation and growth with rich and poor consumers." Metroeconomica 56.2: 233-262. 


\section{Appendix: Not For Publication}

\section{B Appendix - Lower Cutoff}

We here generalize the solution of equilibrium prices (12). Specifically, we assume that the distribution of consumer valuations $(2)$ is uniform on $[\underline{v}, \bar{v}]$, i.e.

$$
G(v)=\left\{\begin{array}{cl}
0 & \text { if } v<\underline{\mathrm{v}} \\
(v-\overline{\mathrm{v}}) /(\underline{\mathrm{v}}-\overline{\mathrm{v}}) & \text { if } v \in[\underline{\mathrm{v}}, \overline{\mathrm{v}}] \\
1 & \text { if } v>\overline{\mathrm{v}}
\end{array}\right.
$$

holds. In addition, we assume that (10) still holds, so that each quality is a constant fraction higher than its immediately preceding one. In this case, the system (7) becomes

$$
p_{n}= \begin{cases}\frac{1}{2}\left[c_{0}+\left(1-\frac{1}{\gamma}\right) q_{0} \bar{v}+p_{-1}\right] & \text { if } n=0 \\ \frac{1}{2}\left[c_{n}+\frac{1}{\gamma+1} p_{n+1}+\frac{\gamma}{\gamma+1} p_{n-1}\right] & \text { if }-N<n<0 \\ \frac{1}{2}\left[c_{-N}+p_{-N+1}-(\gamma-1) q_{-N} \underline{v}\right] & \text { if } n=-N\end{cases}
$$

We can formulate the following proposition (noting that $\left.(\lambda-2 \gamma)=3 \gamma\left(2-\lambda^{-1}\right)^{-1}\right)$.

Proposition 3 Assume equal relative spacing in quality, i.e., (10) holds and marginal production costs are as in (4). Then prices are

$$
p_{n}=\lambda^{n} A_{N}+(\gamma / \lambda)^{N+n} B_{N}+\alpha c_{n} \quad \forall-N \leq n \leq 0
$$

where

$$
\begin{aligned}
\alpha & =\frac{\gamma+1}{2(\gamma+1)-\gamma^{\theta}-\gamma^{1-\theta}} \\
\lambda & =\gamma+1+\sqrt{\gamma^{2}+\gamma+1} \\
A_{N} & =\frac{\lambda-2 \gamma}{3 \gamma} \frac{\gamma a_{0}+(\gamma / \lambda)^{N} b_{N}}{1-\gamma^{N} \lambda^{-2 N}} \\
B_{N} & =\frac{\lambda-2}{3 \gamma} \frac{\lambda^{-N} \gamma a_{0}+b_{N}}{1-\gamma^{N} \lambda^{-2 N}} \\
a_{0} & =\left(1-\left(2-\gamma^{-\theta}\right) \alpha\right) c_{0}+(1-1 / \gamma) q_{0} \bar{v} \\
b_{N} & =\left(1-\left(2-\gamma^{\theta}\right) \alpha\right) c_{-N}-(\gamma-1) q_{-N} \underline{v}
\end{aligned}
$$

and $N$ is defined as

$$
N=\max \left\{m \mid B_{m}+A_{m}\left(\lambda / \gamma^{\theta}\right)^{-m}+\alpha>1\right\} .
$$


Proof. Substitution $u_{n}=p_{n}-\alpha c_{n}$ into (44) yields

$$
2\left[u_{n}+\alpha c_{n}\right]=c_{n}+\frac{1}{\gamma+1}\left[u_{n+1}+\alpha c_{n+1}\right]+\frac{\gamma}{\gamma+1}\left[u_{n-1}+\alpha c_{n-1}\right]
$$

for $n>1$. With $\alpha=(1+\gamma) /\left[2(1+\gamma)-\gamma^{\theta}-\gamma^{1-\theta}\right]$ this is $2(\gamma+1) u_{n}=u_{n+1}+\gamma u_{n-1}$. The equation

$$
X^{2}-2(\gamma+1) X+\gamma=0
$$

has two roots, $\lambda=\left[\gamma+1+\sqrt{\gamma^{2}+\gamma+1}\right]$ larger than unity and $\mu=\left[\gamma+1-\sqrt{\gamma^{2}+\gamma+1}\right]$, smaller than unity. The general solution to the second-order recursive series is thus

$$
p_{n}=\tilde{A} \lambda^{n}+\tilde{B} \mu^{n}+\alpha c_{n}
$$

Equation (44) for $n=0$ is $2 p_{0}=c_{0}+\left(q_{0}-q_{-1}\right) \overline{\mathrm{v}}+p_{-1}$ and, in combination with equation (53) for $n=-1$ implies

$$
2\left(\tilde{A}+\tilde{B}+\alpha c_{0}\right)=c_{0}+\left(q_{0}-q_{-1}\right) \bar{v}+\left(\tilde{A} \lambda^{-1}+\tilde{B} \mu^{-1}+\alpha c_{-1}\right)
$$

while equation (44) for $n=-N$ is $2 p_{-N}=c_{-N}+p_{-N+1}-(\gamma-1) q_{-N} \underline{v}$, in combination with equation (53) for $n=-N+1$ hence implying that

$$
2\left(\tilde{A} \lambda^{-N}+\tilde{B} \mu^{-N}+\alpha c_{-N}\right)=\left(c_{-N}+\tilde{A} \lambda^{-N+1}+\tilde{B} \mu^{-N+1}+\alpha c_{-N+1}-(\gamma-1) q_{-N} \underline{v}\right)
$$

We can substitute $\mu$ using $\lambda \mu=\gamma$, which holds the solution by $\lambda^{2}-2(\gamma+1) \lambda+\gamma=0$. Finally, solving for $\tilde{A}$ and $\tilde{B}$, replacing $A=\tilde{A}$ and $B=\tilde{B} \mu^{-N}$ proves (15).

Corollary 2 Assume that (10) holds. Then,

(i) prices $p_{n}$, markups $p_{n} / c_{n}$ and the slope of the pricing schedule $\left(p_{n+1}-p_{n}\right)$ are increasing in $\bar{v}$ and decreasing in $\underline{v}$ for all $n$.

(ii) if $\gamma^{\theta} \leq \lambda$, the slope of the pricing schedule $\left(p_{n+1}-p_{n}\right)$ is increasing in $\varphi$ for all $n$.

(iii) if $\gamma^{\theta} \leq \lambda$, the slope of the pricing schedule $\left(p_{n+1}-p_{n}\right)$ is increasing in $q_{0}$ for all $n$.

(iv) if $\bar{v}>\underline{v}(\lambda / \gamma)^{N}$ holds, markups $p_{n} / c_{n}$ are decreasing in $\varphi$ for all $n$.

Proof. (i) The statement for prices $p_{n}$ follows since $\gamma>1$ implies with (50) and (51) $d a_{N} / d \bar{v}>0, d a_{N} / d \underline{v}=d b_{N} / d \bar{v}=0$ and $d b_{N} / d \underline{v}<0$ so that with (45) - (51) $d p_{n} / d \bar{v}>0$ 
and $d p_{n} / d \underline{v}<0$. The statement for markups $p_{n} / c_{n}$ follows since $d c_{n} / d \bar{v}=d c_{n} / d \underline{v}=0$.

For the statement regarding $\left(p_{n+1}-p_{n}\right)$ compute with (45)

$$
\frac{d\left(p_{n+1}-p_{n}\right)}{d \bar{v}}=(\lambda-1) \lambda^{n} \frac{d A_{N}}{d \bar{v}}+(\gamma / \lambda-1)(\gamma / \lambda)^{N+n} \frac{d B_{N}}{d \bar{v}}
$$

Using (48) - (51), check that

$$
\frac{d A_{N}}{d \bar{v}}=\frac{\lambda-2 \gamma}{3 \gamma} \frac{\gamma}{1-\left(\gamma / \lambda^{2}\right)^{N}}(1-1 / \gamma) q_{0} \quad \text { and } \quad \frac{d B_{N}}{d \bar{v}}=\frac{\lambda-2}{3 \gamma} \frac{\lambda^{-N} \gamma}{1-\left(\gamma / \lambda^{2}\right)^{N}}(1-1 / \gamma) q_{0}
$$

By $\gamma / \lambda^{2}<1, d\left(p_{n+1}-p_{n}\right) / d \bar{v}$ is positive if and only if

$$
[\lambda-1][\lambda-2 \gamma] \lambda^{n}-[1-\gamma / \lambda][\lambda-2](\gamma / \lambda)^{N+n} \lambda^{-N}
$$

is positive. Since $\lambda>2 \gamma$, all terms in squared brackets are positive so that the expression above is increasing in $n$ and hence minimal for $n=-N$. It is thus sufficient to show that

$$
[\lambda-1][\lambda-2 \gamma]-[1-\gamma / \lambda][\lambda-2]>0
$$

holds. But this inequality is satisfied since $\lambda$ solves the identity $\lambda^{2}-(2 \gamma+1) \lambda+2 \gamma=0$ (see (52) in the proof of (45) in the appendix) so that the expression above equals

$$
2 \gamma+2-2 \gamma / \lambda>0
$$

This proves that $d\left(p_{n+1}-p_{n}\right) / d \bar{v}>0$.

Next, compute

$$
\frac{d\left(p_{n+1}-p_{n}\right)}{d \underline{v}}=(\lambda-1) \lambda^{n} \frac{d A_{N}}{d \underline{v}}+(\gamma / \lambda-1)(\gamma / \lambda)^{N+n} \frac{d B_{N}}{d \underline{v}}
$$

Using (48) - (51), check that

$$
\frac{d A_{N}}{d \underline{\bar{v}}}=\frac{\lambda-2 \gamma}{3 \gamma} \frac{-(\gamma / \lambda)^{N}}{1-\gamma^{N} \lambda^{-2 N}}(\gamma-1) q_{-N} \text { and } \frac{d B_{N}}{d \underline{v}}=\frac{\lambda-2}{3 \gamma} \frac{-1}{1-\gamma^{N} \lambda^{-2 N}}(\gamma-1) q_{-N}
$$

so that $d\left(p_{n+1}-p_{n}\right) / d \underline{v}$ is negative if and only if

$$
-[\lambda-1][\lambda-2 \gamma]+[1-\gamma / \lambda][\lambda-2]\left(\gamma / \lambda^{2}\right)^{n}<0
$$

holds. Since all terms in squared brackets are positive and $\gamma / \lambda^{2}<1$ this expression is maximal at $n=0$ so that the statement holds by (51) above.

(ii) First notice with (46), (50) and (51) that

$$
a_{0}=-\beta c_{0}+(1-1 / \gamma) q_{0} \bar{v} \quad \text { and } \quad b_{N}=\gamma \beta c_{-N}-(\gamma-1) q_{-N} \underline{v}
$$


where

$$
\beta=\frac{\gamma^{\theta}-\gamma^{-\theta}}{2(\gamma+1)-\gamma^{\theta}-\gamma^{1-\theta}}
$$

and compute with (45) - (51)

$$
\begin{aligned}
\frac{d\left(p_{n+1}-p_{n}\right)}{d \varphi}= & \lambda^{n}(\lambda-1) \frac{\partial A_{N}}{\partial \varphi}-\left(\frac{\gamma}{\lambda}\right)^{N+n}\left(1-\frac{\gamma}{\lambda}\right) \frac{\partial B_{N}}{\partial \varphi}+\gamma^{\theta n} \alpha\left(\gamma^{\theta}-1\right) \frac{c_{0}}{\varphi} \\
= & {\left[-\left(\lambda^{n}(\lambda-1)(\lambda-2 \gamma) \frac{1-\left(\frac{\gamma^{1-\theta}}{\lambda}\right)^{N}}{1-\left(\frac{\gamma}{\lambda^{2}}\right)^{N}}+\ldots\right.\right.} \\
& \left.\left.\ldots+\left(\frac{\gamma}{\lambda}\right)^{N+n}(1-\gamma / \lambda)(\lambda-2) \lambda^{-N} \frac{\left(\frac{\gamma^{\theta}}{\lambda}\right)^{-N}-1}{1-\left(\frac{\gamma}{\lambda^{2}}\right)^{N}}\right) \frac{\beta}{3}+\gamma^{\theta n} \alpha\left(\gamma^{\theta}-1\right)\right] \frac{c_{0}}{\varphi}
\end{aligned}
$$

Using $\lambda^{2}=2(\gamma+1) \lambda-\gamma$ from (52) we can substitute

$$
(\lambda-1)(\lambda-2 \gamma)=\lambda+\gamma \text { and }(1-\gamma / \lambda)(\lambda-2)=\gamma \frac{\lambda+1}{\lambda}
$$

so that

$$
\begin{aligned}
\frac{d\left(p_{n+1}-p_{n}\right)}{d \varphi}= & {\left[-\left((\lambda+\gamma) \frac{1-\left(\frac{\gamma^{1-\theta}}{\lambda}\right)^{N}}{1-\left(\frac{\gamma}{\lambda^{2}}\right)^{N}}+\ldots\right.\right.} \\
& \left.\left.\ldots+\left(\frac{\gamma}{\lambda^{2}}\right)^{n}\left(\frac{\gamma}{\lambda}\right)^{N} \gamma \frac{\lambda+1}{\lambda} \lambda^{-N} \frac{\left(\frac{\gamma^{\theta}}{\lambda}\right)^{-N}-1}{1-\left(\frac{\gamma}{\lambda^{2}}\right)^{N}}\right) \frac{\beta}{3}+\left(\frac{\gamma^{\theta}}{\lambda}\right)^{n} \alpha\left(\gamma^{\theta}-1\right)\right] \lambda^{n} \frac{c_{0}}{\varphi}
\end{aligned}
$$

To prove the claim, we will show that the expression in square brackets is positive. As a first step, we look at the terms that depend on $n$ :

$$
\left(\frac{\gamma}{\lambda^{2}}\right)^{n}\left[-\gamma \frac{\lambda+1}{\lambda}\left(\frac{\gamma}{\lambda^{2}}\right)^{N} \frac{\left(\frac{\gamma^{\theta}}{\lambda}\right)^{-N}-1}{1-\left(\frac{\gamma}{\lambda^{2}}\right)^{N}}\left(\gamma^{\theta}-\gamma^{-\theta}\right)\right]+\left(\frac{\gamma^{\theta}}{\lambda}\right)^{n}\left[3(\gamma+1)\left(\gamma^{\theta}-1\right)\right]
$$

at $n=-N$ this expression equals

$$
\begin{aligned}
\ldots & =-\gamma \frac{\lambda+1}{\lambda} \frac{\left(\lambda / \gamma^{\theta}\right)^{N}-1}{1-\gamma^{N} \lambda^{-2 N}}\left(\gamma^{\theta}-\gamma^{-\theta}\right)+3\left(\frac{\gamma^{\theta}}{\lambda}\right)^{-N}(\gamma+1)\left(\gamma^{\theta}-1\right) \\
& =\left[-\gamma \frac{\lambda+1}{\lambda} \frac{1-\left(\lambda / \gamma^{\theta}\right)^{-N}}{1-\gamma^{N} \lambda^{-2 N}} \frac{\gamma^{\theta}-\gamma^{-\theta}}{\gamma^{\theta}-1}+3(\gamma+1)\right]\left(\frac{\gamma^{\theta}}{\lambda}\right)^{-N}\left(\gamma^{\theta}-1\right)
\end{aligned}
$$


The expression in square brackets satisfies

$$
\begin{aligned}
-\gamma \frac{\lambda+1}{\lambda} \frac{1-\left(\gamma^{\theta} / \lambda\right)^{N}}{1-\left(\gamma / \lambda^{2}\right)^{N}} \frac{\gamma^{\theta}-\gamma^{-\theta}}{\gamma^{\theta}-1}+3(\gamma+1) & >-\gamma \frac{\lambda+1}{\lambda} \frac{\gamma^{\theta}-\gamma^{-\theta}}{\gamma^{\theta}-1}+3(\gamma+1) \\
& >-\gamma \frac{\lambda+1}{\lambda} 2+3(\gamma+1)>0
\end{aligned}
$$

Thus we have shown that, in absolute terms, the positive expression in the second squared brackets in (56) is larger than the negative term in the first. Thus, multiplying the negative term by $\left(\gamma / \lambda^{2}\right)$ and the positive term by $\left(\gamma^{\theta} / \lambda\right)$ increases the sum of both. Hence the expression in (56) is increasing in $n$ and so is the expression in (55). Therefore it is sufficient to show that the expression in (55) is positive for $n=-N$. To this aim, we set $n=-N$ in (55), multiply by $\left(\gamma^{\theta} / \lambda\right)^{N}$ and show next that

$$
-\left((\lambda+\gamma) \frac{\left(\frac{\gamma^{\theta}}{\lambda}\right)^{N}-\left(\frac{\gamma}{\lambda^{2}}\right)^{N}}{1-\left(\frac{\gamma}{\lambda^{2}}\right)^{N}}+\gamma \frac{\lambda+1}{\lambda} \frac{1-\left(\frac{\gamma^{\theta}}{\lambda}\right)^{N}}{1-\left(\frac{\gamma}{\lambda^{2}}\right)^{N}}\right)\left(\gamma^{\theta}-\gamma^{-\theta}\right)+3(\gamma+1)\left(\gamma^{\theta}-1\right)
$$

is positive. To do so, we use that $\frac{\left(\gamma^{\theta} / \lambda\right)^{N}-\gamma^{N} \lambda^{-2 N}}{1-\gamma^{N} \lambda^{-2 N}}+\frac{1-\left(\gamma^{\theta} / \lambda\right)^{N}}{1-\gamma^{N} \lambda^{-2 N}}=1$ so that we can rewrite the expression above as

$$
\left(\frac{1-\left(\gamma^{\theta} / \lambda\right)^{N}}{1-\gamma^{N} \lambda^{-2 N}}\left((\lambda+\gamma)-\gamma \frac{\lambda+1}{\lambda}\right)-(\lambda+\gamma)\right)\left(\gamma^{\theta}-\gamma^{-\theta}\right)+3(1+\gamma)\left(\gamma^{\theta}-1\right)
$$

Using now that $\frac{1-\left(\gamma^{\theta} / \lambda\right)^{N}}{1-\gamma^{N} \lambda^{-2 N}}<1$ is increasing in $N$ and $N \geq 1$, we have

$$
\begin{aligned}
\ldots & \geq\left(\frac{1-\gamma^{\theta} / \lambda}{1-\gamma / \lambda^{2}}\left((\lambda+\gamma)-\gamma \frac{\lambda+1}{\lambda}\right)-(\lambda+\gamma)\right)\left(\gamma^{\theta}-\gamma^{-\theta}\right)+3(1+\gamma)\left(\gamma^{\theta}-1\right) \\
& =-\left(\gamma^{\theta}+\gamma\right)\left(\gamma^{\theta}-\gamma^{-\theta}\right)+3(1+\gamma)\left(\gamma^{\theta}-1\right)
\end{aligned}
$$

To check that this last expression is positive, we divide by $(1+\gamma)\left(\gamma^{\theta}-1\right)$ and look at

$$
-\frac{\left(\gamma^{\theta}+\gamma\right)}{(1+\gamma)} \frac{\left(\gamma^{\theta}-\gamma^{-\theta}\right)}{\left(\gamma^{\theta}-1\right)}+3 \geq-\frac{(\lambda+\gamma)}{(1+\gamma)} \frac{(\lambda-1 / \lambda)}{(\lambda-1)}+3
$$

where we have used $\gamma^{\theta} \leq \lambda$. Simplifying further with $\lambda^{2}=2(\gamma+1) \lambda-\gamma$ from (52) leads to

$$
\begin{aligned}
-\frac{(\lambda+\gamma)}{(1+\gamma)} \frac{(\lambda-1 / \lambda)}{(\lambda-1)}+3 & =-\frac{\lambda^{2}-1+\gamma \lambda-\gamma / \lambda}{(1+\gamma)(\lambda-1)}+3 \\
& =-\frac{2(\gamma+1) \lambda-(\gamma+1)}{(1+\gamma)(\lambda-1)}-\frac{\lambda-1 / \lambda}{(1+\gamma)(\lambda-1)}+3 \\
& >-\frac{2 \lambda-1}{\lambda-1}-\frac{\gamma \lambda}{\gamma \lambda+\lambda-(\gamma+1)}+3>-2-1+3=0
\end{aligned}
$$


The last inequality holds by $\lambda>(\gamma+1)$ and proves the statement.

(iii) Notice with (45) - (51) that

$$
\frac{d}{d q_{0}}\left(\frac{p_{n+1}-p_{n}}{q_{0}}\right)=\frac{d\left(p_{n+1}-p_{n}\right)}{d \varphi}\left(\frac{\varphi}{q_{0}^{2}} \frac{\theta-1}{\theta}\right)
$$

Since furthermore,

$$
\frac{d}{d q_{0}}\left(\frac{p_{n+1}-p_{n}}{q_{0}}\right)=\frac{1}{q_{0}} \frac{d\left(p_{n+1}-p_{n}\right)}{d q_{0}}-\frac{p_{n+1}-p_{n}}{q_{0}^{2}}
$$

we have

$$
\begin{aligned}
\frac{d\left(p_{n+1}-p_{n}\right)}{d q_{0}} & =q_{0} \frac{d}{d q_{0}}\left(\frac{p_{n+1}-p_{n}}{q_{0}}\right)+\frac{p_{n+1}-p_{n}}{q_{0}} \\
& =q_{0} \frac{d\left(p_{n+1}-p_{n}\right)}{d \varphi}\left(\frac{\varphi}{q_{0}^{2}} \frac{\theta-1}{\theta}\right)+\frac{p_{n+1}-p_{n}}{q_{0}}
\end{aligned}
$$

which is positive by (ii).

(iv) Compute with (45) - (51)

$$
\begin{aligned}
& \frac{d\left(p_{n} / c_{n}\right)}{d \varphi}= \lambda^{n} \frac{d\left(A_{N} / c_{n}\right)}{d \varphi}+(\gamma / \lambda)^{N+n} \frac{d\left(B_{N} / c_{n}\right)}{d \varphi} \\
&= \frac{1}{3 \gamma\left(1-\gamma^{N} \lambda^{-2 N}\right)}\left\{\lambda^{n}(\lambda-2 \gamma)\left[\gamma \frac{d\left(a_{0} / c_{n}\right)}{d \varphi}+(\gamma / \lambda)^{N} \frac{d\left(b_{N} / c_{n}\right)}{d \varphi}\right]+\ldots\right. \\
&\left.\ldots+(\gamma / \lambda)^{N+n}(\lambda-2)\left[\lambda^{-N} \gamma \frac{d\left(a_{0} / c_{n}\right)}{d \varphi}+\frac{d\left(b_{N} / c_{n}\right)}{d \varphi}\right]\right\} \\
&= \frac{(\gamma-1) /\left(\varphi c_{n}\right)}{3 \gamma\left(1-\gamma^{N} \lambda^{-2 N}\right)}\left\{\lambda^{n}(\lambda-2 \gamma)\left[-q_{0} \bar{v}+(\gamma / \lambda)^{N} q_{-N} \underline{v}\right]+\ldots\right. \\
&\left.\ldots+(\gamma / \lambda)^{N+n}(\lambda-2)\left[-\lambda^{-N} q_{0} \bar{v}+q_{-N} \underline{v}\right]\right\}
\end{aligned}
$$

Straight forward simplification with $q_{-N}=\gamma^{-N} q_{0}$ leads to

$$
\frac{d\left(p_{n} / c_{n}\right)}{d \varphi}=\frac{q_{0} \underline{v}(\gamma-1) /\left(\varphi c_{n}\right)}{3 \gamma\left(1-\gamma^{N} \lambda^{-2 N}\right)}\left\{\lambda^{n}(\lambda-2 \gamma)\left[-\frac{\bar{v}}{\underline{v}}+\lambda^{-N}\right]+(\gamma / \lambda)^{N+n}(\lambda-2)\left[-\lambda^{-N} \frac{\bar{v}}{\underline{v}}+\gamma^{-N}\right]\right\}
$$

Since $\bar{v} / \underline{v}>1>\lambda^{-N}$ holds always and $\bar{v} / \underline{v}>(\lambda / \gamma)^{N}$ by assumption, the term on the right is negative, which proves the statement. 